\title{
Comparative Study between Emulsification and Microfluidic Techniques for Bovine Serum Albumin loaded Biodegradable Nanoparticles.
}
Omar ma'ayah ${ }^{1}$, Nazim Uddinn ${ }^{2}$, Samuel Girgis ${ }^{3}$
${ }^{1}$ School of pharmacy and pharmaceutical sciences, University of Sunderland
University Department,
Sunderland, State SR1 3SD, United Kingdom
maayah.omar1@gmail.com, 00447425215850. \\ ${ }^{2}$ School of pharmacy and pharmaceutical sciences, University of Sunderland \\ University Department, \\ Sunderland, State SR1 3SD, United Kingdom \\ bg65zj@ research.sunderland.ac.uk, 00447824809123. \\ ${ }^{3}$ School of pharmacy and pharmaceutical sciences, University of Sunderland \\ University Department, \\ Sunderland, State SR1 3SD, United Kingdom \\ bg96cd@research.sunderland.ac.uk, 00447404780179
}

The aim of this study was to investigate the nanoparticles prepared either by conventional techniques including double emulsion method/ solvent evaporation, or by novel approaches providing an optimal therapeutic efficacy for pharmaceutical applications such as microfluidic technique. BSA (Bovine Serum Albumin) was used as a drug model, which is characterised as a peptide. The performance of nanoparticles was analysed with characterisations such as size, Zeta, Polydespersity Index (PDI), therapeutic efficiency, in vitro release study, Scanning Electron Microscopy (SEM), and Fourier Transform Infrared Spectrophotometer (FTIR). In this study, two formulations were prepared for each method; double emulsion and microfluidic techniques. The formulations involved PLGA polymer and PCL polymer. Each of those formulations were triplicated to provide accurate data for the investigation. All formulations were examined in Nano size range (less than $1 \mu \mathrm{m})$ with negative zeta potential values between -7 and $-8 \mathrm{mV}$. Although, all BSA nanoparticles were prepared in a spherical shape to maximise the entrapment efficiency. Yet, the nanoparticles prepared by microfluidic technique had lower PDI than those prepared by double emulsion technique. Additionally, the nanoparticles prepared by microfluidic technique had higher encapsulation efficiency than those prepared by double emulsion method. This study would be applicable for further researches, since BSA nanoparticles could be designed as a targeted delivery system which provides controlled release with high encapsulation efficiency.

Key words: Double emulsion, Solvent evaporation, Microfluidics, Bovine serum albumin, PLGA, PCL.

Corresponding Author: Samuel Girgis 


\section{INTRODUCTION}

The encapsulation of therapeutic peptides and proteins inside biodegradable nanoparticles was commonly utilised to protect against harsh conditions for instance: $\mathrm{pH}$, temperature, and proteolysis (Martinez et al., 2017). The major obstacle related to peptide drugs is the instability in acidic pH environment, due to the presence of digestive enzymes in the stomach namely pepsin. Moreover, the physicochemical properties of peptides play a fundamental role on the therapeutic efficiency. Among those peptides, is insulin which is characterised with high molecular weight and poor diffusion through the mucous layer in the gastrointestinal tract. As a result, those characters would hinder the bioavailability of insulin (Morishita and Peppas, 2006). Nowadays, the advantages acquainted with using biodegradable nanoparticles involve targeting specific organs, providing more efficient delivery, and reducing the side effects (Kumari, Yadav and Yadav, 2010). Polymeric nanoparticles are used to improve patient's compliance as in treating diabetic mellitus patients (Iqbal et al., 2015). The aim of this research is to design novel nanoparticles using biodegradable polymers to provide a sustained release of therapeutic peptides as Insulin. Those particles would therefore reduce the dosing frequency and hence efficiency.

Poly (D,L-lactic-co-glycolic acid) PLGA, which is commonly used in pharmaceutical industry and often modified with poly (ethylene glycol) PEG, composed of two polyesters Polylactic acid (PLA) and Polyglycolic acid (PGA) through ring opening polymerization, as seen in figure (1) (Lassalle and Ferreira, 2010). PLGA with the molecular weight of 24-38 $\mathrm{kDa}$ has a negative charge. Because of its biocompatible and biodegradable properties, PLGA is approved by FDA (Iqbal et al., 2015). PEG could be used with PLGA as a block co-polymer to enhance drug encapsulation (Locatelli and Comes Franchini, 2012). Several therapeutic proteins and peptides were encapsulated successfully inside PLGA nanoparticles such as insulin loaded PLGA nanoparticles. According to Haggag study, the in vitro release of insulin got improved when insulin was encapsulated inside PLGA polymer (Haggag et al., 2016).

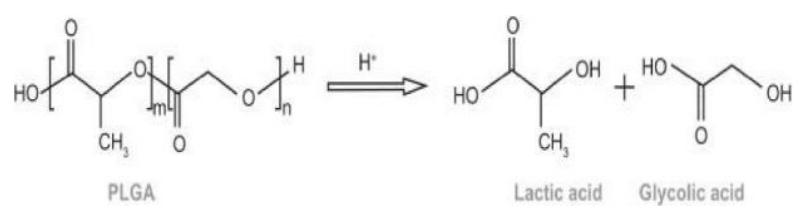

\section{Figure (1): showing the degradation of polylactide-co-glycolide to lactic and glycolic acid (Jain, 2000).}

Poly ( $\varepsilon$-caprolactone) PCL, which made of a cyclic ester and $\varepsilon$-caprolactone, is synthesized by ring opening followed by the polymerization of $\varepsilon$-caprolactone under high temperature conditions $\left(140-150^{\circ} \mathrm{C}\right)$, as seen in figure (2). Additionally, PCL is known to have the lowest melting point; $60^{\circ} \mathrm{C}$ amongst biodegradable polymers (Abdellah Ali, 2016). Further, PCL has biocompatible properties in terms of the susceptibility of its aliphatic ester linkage to be hydrolysed (Tavares et al., 2017). Due to its high hydrophobicity, PCL is absorbed from the mucosal layer in stomach.

Several therapeutic proteins and peptides incorporated successfully inside PCL nanoparticles, showed an enhanced therapeutic effect. For example, tamoxifen is a nonsteroidal agent and anti-estrogenic drug to prevent the proliferation of cancer cells. The encapsulation of tamoxifen in PCL nanoparticles showed significant accumulation as well as more extended release towards the tumor cells (Kumari, Yadav and Yadav, 2010). Several techniques were used to encapsulate peptides in biodegradable polymers such as nanoprecipitation, coacervation, single emulsion, double emulsion evaporation, and microfluidic techniques (Cohen-Sela et al., 2009). 


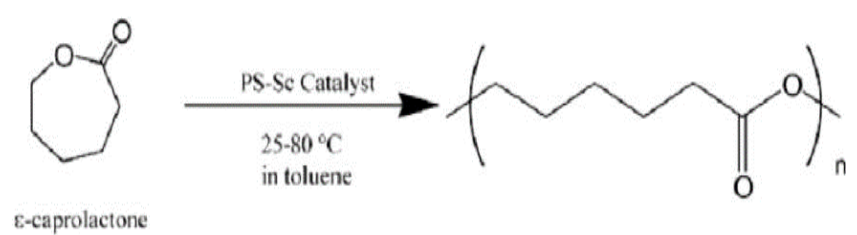

PCL

\section{Figure (2): showing the Ring opening polymerization of \&-caprolactone to polycaprolactone (Woodruff and Hutmacher, 2010).}

One of the most common formulation methods is double emulsion (w/o/w) / solvent evaporation technique. This technique shows more efficiency in encapsulation of hydrophilic drugs compared to other methods. The primary emulsion is prepared using a shear force resulting from the homogenization of a mixture composed of a hydrophilic drug in the inner aqueous phase and a polymer in the organic phase. Then, using the same shear force to make the secondary emulsion by adding an aqueous medium containing a stabiliser (Haggag et al., 2016). Polyvinyl alcohol (PVA) is the most common emulsifier used as a stabilizer for the prepared emulsions. PVA is characterised by forming an interconnected network with polymers. The parameters that influence the properties of nanoparticles prepared by double emulsion technique include the type of organic solvent used in the emulsion and the concentration of PVA (Sahoo et al., 2002). The advantages of the double emulsion technique involve the alteration in the physicochemical properties of peptides nanoparticles such as the release properties, surface charge, encapsulation efficiency and toxic effect of both peptides and proteins. However, the critical limitations acquainted with double emulsion / solvent evaporation technique are summarised in the poor-quality control during nanoparticles preparation, reproducibility problems, and less size homogeneity. Recently, microfluidic technique has become interesting for creating the emulsion droplet with controllable structures. The encapsulation efficiency of drugs into nanoparticles prepared by microfluidic technique was higher than nanoparticles obtained by other techniques. The microfluidic technique provides high bioavailability and pharmacokinetic profile for peptide drugs (Precisionnanosystems.com, 2018). In this method, two solutions are injected through two inlets to create nanoparticles. The first solution contains a polymer solution while the second solution contains an aqueous buffer. Then, two solutions are injected together inside the microchannel and come out through one outlet to create nanoparticles (Chiesa et al., 2018). PLGA is used in the microfluidic technique which therefore allows the emulsion droplets to have more size uniformity and a precise control of the nanoparticles physical. In addition, the microfluidic technique provides the possibility for batch production in shorter time compared to the double emulsion method (Joshi et al., 2016).

The aim of this project is to design formulations that could overcome the major limitations associated with the therapeutic peptides. To develop the physicochemical properties of therapeutic peptides, biodegradable nanoparticles were used to protect the therapeutic peptides from harsh conditions through two techniques double emulsion and microfluidic. In this study, four formulations consisting of BSA loaded PLGA and BSA loaded PCL nanoparticles were prepared using two techniques; double emulsion and microfluidic technique. The formulated nanoparticles were characterised using Zetasizer, zeta potential and polydespirty index (PDI). Besides, the morphological structure was determined, and the encapsulation efficiency was calculated. Further studies were carried out, involving a release study, and FTIR study. This type of system relies on a combined effect of hydration in its two compartments such that, initially, the drug layer draws sufficient water (pull) to form a saturated aqueous suspension or solution, which will be expulsed subsequently through the delivery orifice once the generated osmotic pressure and the swelling of the push layer 1, 2 . 
Among the main advantages of these systems is that drug release is not influenced by physiological conditions, such as stomach $\mathrm{pH}$, peristaltic movements and food contents in the gastrointestinal tract. Furthermore, the systems exhibit a high grade of in-vitro and in-vivo correlation 3. However, despite these advantages, these systems have certain limitations in terms of release rates of drugs with solubility lower than $0.05 \mathrm{~g} / \mathrm{mL}$ or practically insoluble.

\section{MATERIALS AND METHODS}

\subsection{Materials.}

Albumin from bovine serum minimum 98\% electrophoresis with MW $66 \mathrm{kDa}$, PLGA (Resomer®RG 503, Poly (DL-lactide-co- glycolide MW 24,000-35,000 lactic: glycolide 50:50), copolymer of Poly (DL-Lactide-co-caprolactone with $86 \%$ mol), Poly (vinyl alcohol Mw 31,000-50,000, 87\%-89\% hydrolysed), potassium chloride ( $\mathrm{KCl})$, Sodium dodecyl sulfate $(\mathrm{SDS})$, sodium hydroxide $(\mathrm{NaOH})$, and hydrochloric acid $(\mathrm{HCl})$ were all purchased from Sigma Aldich ${ }^{\circledR}$, UK. Micro BCATM Protein Assay Kit was obtained from Pierce (Thermoscintific). PierceTM Acetonitrile (ACN) and dichloromethane (DCM) were of LC-MS Grade. Table (1): showing different types of formulation prepared by different types of polymers and techniques.

Table (1): different types of formulation

\begin{tabular}{|c|c|c|c|c|c|}
\hline $\begin{array}{l}\text { Formulation } \\
\text { ID }\end{array}$ & Technique & $\begin{array}{l}\text { Poly (DL- } \\
\text { lactide-co- } \\
\text { glycolide } \%\end{array}$ & $\begin{array}{c}\text { Poly (D, L- } \\
\text { lactide-co- } \\
\text { caprolactone) } \\
\%\end{array}$ & $\begin{array}{l}\text { DL- } \\
\text { Lactide } \\
\%\end{array}$ & $\begin{array}{l}\text { Bovine serum } \\
\text { albumin(mg) }\end{array}$ \\
\hline 1'LGAA OI & \multirow{6}{*}{$\begin{array}{c}\text { Double } \\
\text { emulsion } \\
\text { solvent } \\
\text { evaporation }\end{array}$} & 50 & 50 & 0 & 5 \\
\hline PICGA 02 & & 50 & 50 & 0 & 5 \\
\hline PLCHA 03 & & 50 & 50 & 0 & 5 \\
\hline PCI, 01 & & 0 & 85 & 15 & 5 \\
\hline PCI. 02 & & 0 & 85 & 15 & 5 \\
\hline PCL 03 & & 0 & 85 & 15 & 5 \\
\hline PI C.A 01 & \multirow{6}{*}{$\begin{array}{c}\text { Microfluidic } \\
\text { technique }\end{array}$} & 50 & 50 & 0 & 5 \\
\hline PLGAO2 & & 50 & 50 & 0 & 5 \\
\hline PLGA 03 & & 50 & 50 & 0 & 5 \\
\hline PCL 01 & & 0 & 85 & 15 & 5 \\
\hline PCL 02 & & 0 & 85 & 15 & 5 \\
\hline PCI. 03 & & 0 & 85 & 15 & 5 \\
\hline
\end{tabular}




\subsection{Methods.}

2.2.1. Preparation of nanoparticles via Double emulsion technique w/o/w.

An internal aqueous bovine serum albumin solution $(5 \mathrm{mg}$ of bovine serum albumin was dissolved in $1 \mathrm{~mL}$ of PVA $2.5 \%$ using vortex, whirl mixer®) was added drop-wise to an organic phase (100 mg of polymers was dissolved in $10 \mathrm{~mL}$ of dichloromethane). The primary emulsion was made using a low speed homogeniser (sturart ${ }^{\circledR}$, homogeniser SHM2) in an ice bath for 2 minutes at $1000 \mathrm{rpm}$. Then, the primary emulsion was added dropwise to the 50 mL PVA $1.25 \%$ in an ice bath using a high-speed homogeniser (Silverson L5T Homogeniser, Silverson Machines, England) for 6 minutes at 10,000 rpm as shown in figure (3). The secondary emulsion was stirred on a magnetic stirrer for overnight ( 24 hours) in the fume hood to allow the evaporation of dichloromethane. Nanoparticles were collected by centrifugation for 30 minutes at $4^{\circ} \mathrm{C}$ using (blackman ${ }^{\circledR}$, model j2-21 centrifuge) and washed with distilled water three times. Nanoparticle samples were kept in the freezer at $-80{ }^{\circ} \mathrm{C}$ for 2 hours and then freeze-dried on ALPHA2-4 LSC CHRIST® for 48 hours.

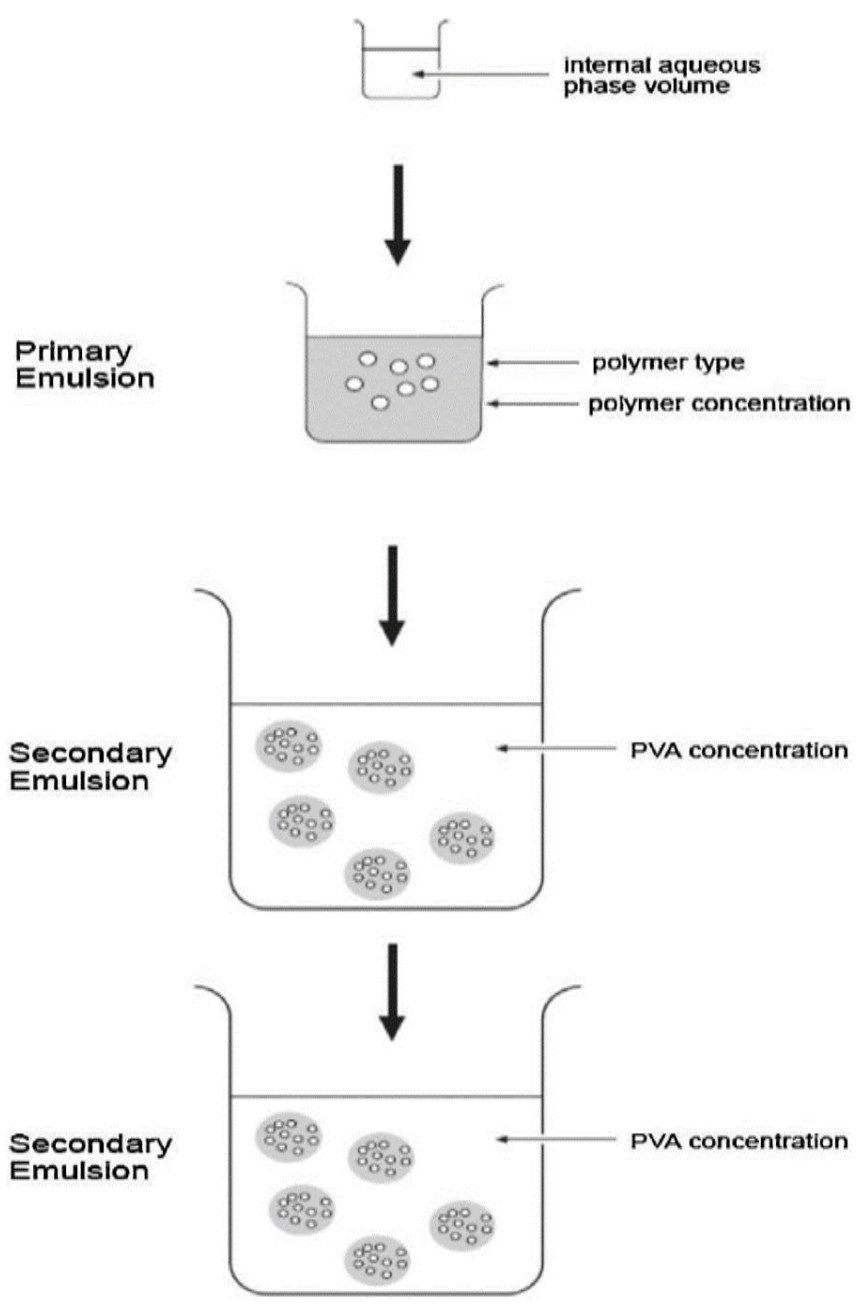

Figure (3): showing the preparation process of BSA NP prepared by double emulsion technique (Haggag et al., 2016). 
2.2.2. Preparation of nanoparticles via Microfluidics technique.

$100 \mathrm{mg}$ of polymers (PLGA, PCL) were dissolved into $4 \mathrm{~mL}$ of acetonitrile and then injected into the first inlet and an aqueous buffer $(5 \mathrm{mg}$ of BSA was dissolved into $1 \mathrm{~mL}$ of PVA $2.5 \%$ ) was injected into the second inlet into NanoAssemblrTM. The nanoparticles were coming out from one outlet, as seen in figure (4). The nanoparticles samples were stirred on a magnetic stirrer for overnight in the fume hood and followed by centrifugation for 30 minutes at $4^{\circ} \mathrm{C}$ using (blackman ${ }^{\circ}$, model j2-21 centrifuge) and washed three times with distilled water. Nanoparticles samples were kept in the freezer at $-80^{\circ} \mathrm{C}$ for 2 hours and then freezedried on for 48 hours.

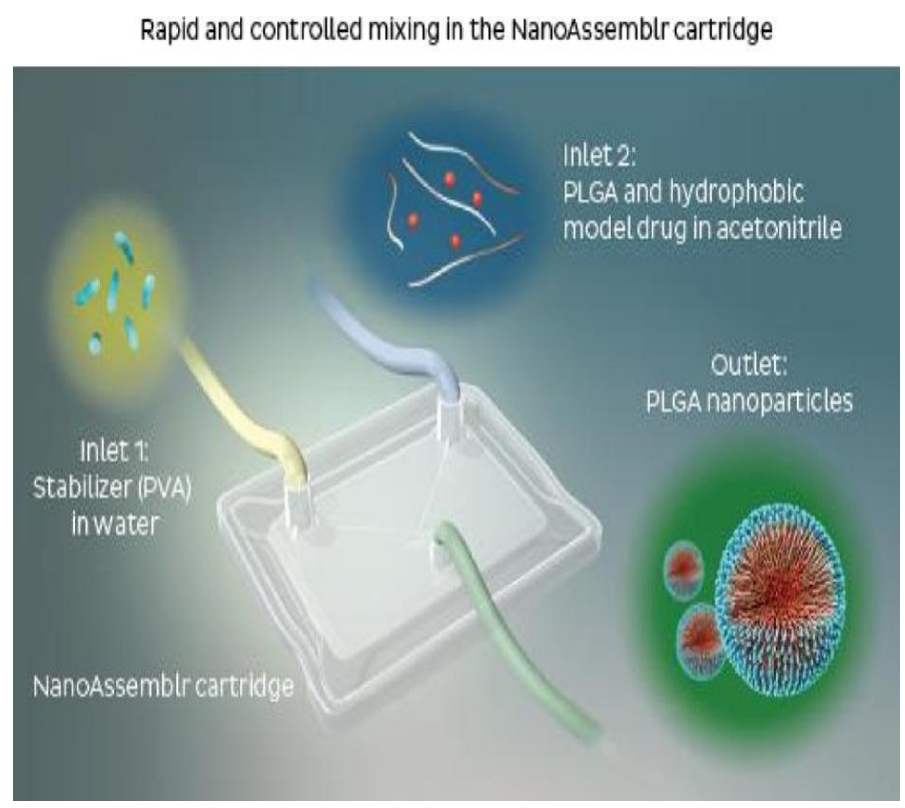

Figure (4): showing the preparation process BSA NP prepared by microfluidic technique (Precisionnanosystems.com, 2018).

\section{Nanoparticle characterizations.}

\subsection{Particles size.}

$5 \mathrm{mg}$ of nanoparticles of each sample were diluted with distilled water and suspended for 2 minutes using vortex mixer. The mean size distribution and PDI were analysed by photon correlation spectroscopy (PCS) using a Malvern Zetasizer ${ }^{\circledR} 5000$ (Malvern Instruments, UK). All measurements were performed in triplicates. The principle of particle size is a direct relationship between the size of the particles and their speed, due to Brownian motion, which is defined in the Stokes-Einstein equation. Brownian motion is the movement of particles, due to the random collisions with the molecules of the liquid that surround the particle. An important feature of Brownian motion is that small particles move quickly and large particles move more slowly. As shown in figure (5), by illuminating the particles with a laser, the speckle pattern produced. By specific angle with the scattering intensity will flocculate and then can be detected using a sensitive avalanche photodiode detector (APD). By changing the intensity followed by the analysis using a digital, which provide a correlation function. The curve produced and analysed to show the particle size distribution. Zetasizer Nano ZS® is manageable with high sensitive performance. Samples were measured in small amounts range with little or without dilution. Zetasizer is suitable to measure from nanoparticles to emulsion and macromolecules (Malvern.com, 2017). 


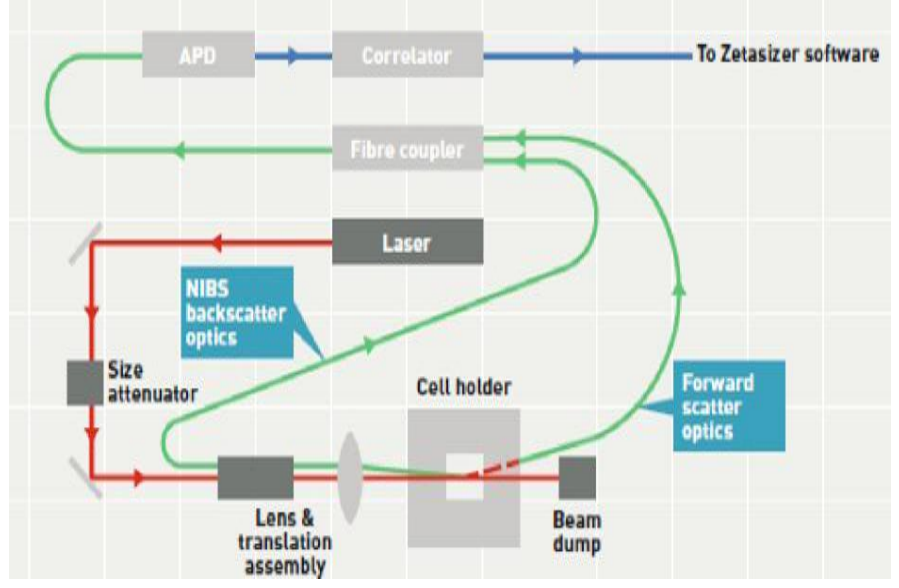

Figure (5): showing the principle of Zetasizer Nano (Malvern.com, 2017).

\subsection{Zeta potential.}

$0.2 \mathrm{ml}$ of samples were dissolved in $0.8 \mathrm{ml}$ of $0.001 \mathrm{M} \mathrm{KCl}$. The zeta potential was analysed by using Zetasizer Nano ZS (Malvern Zetasizer 5000, Malvern Instruments, UK). Samples were distilled using $0.001 \mathrm{M} \mathrm{KCl}$ to improve the sensitivity and accuracy of the measurements. Zeta potential is the charge acquired from the particles's surface or can be defined as the charge between nanoparticles's surface and the outside medium (electrical double layer), as explained in the figure (6). The principle of zeta potential measurement is when the electric source is applied to the sample, the particles start moving because of the interaction between the particles' charge and the electric field and then measured by using the patented laser interferometric technique calls M3-PALS (phase analysis light scattering). This technique provides a mean zeta potential. Eventually, the value of zeta potential gives more details about the state of surface, velocity, and stability of nanoparticles.

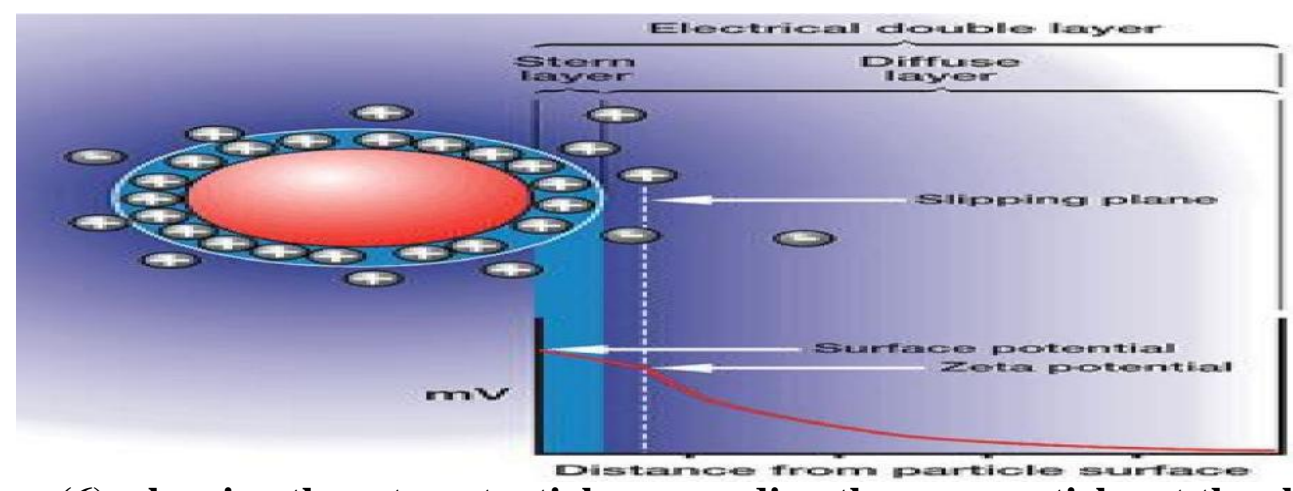

Figure (6): showing the zeta potential surrounding the nanoparticles at the electrical double layer (Waterworld.com, 2018).

\subsection{Morphology study.}

$5 \mathrm{mg}$ of nanoparticles samples were examined using scanning electron microscopy after coating with a mixture of gold and palladium under vacuum using (Quorum® ${ }^{\circledR}$ SC7620 Sputter Coater) in order to improve the quality of images. Then the images were taken using scanning electron microscopy (S-3000N, Hitachi®, Japan), which provides the samples' image up to 50,000x. Scanning electron microscopy (SEM) is a useful technique to provide details about the structure and shape of nanoparticles. SEM uses an electron beam, which applies on the surface of sample. The interactions between the samples and electron beams 
cause the images. (Engineered Nanoparticles: Structure, Properties and Mechanisms of Toxicity Ashok K. Singh, 2017).

\subsection{Determination of bovine serum albumin loading and encapsulation efficiency (EE) by BCA.}

$5 \mathrm{mg}$ of BSA NPs were measured in Eppendorf tubes and then dissolved into $1 \mathrm{~mL}$ of $1 \mathrm{M}$ sodium hydroxide $(\mathrm{NaOH})$ and incubated at $37^{\circ} \mathrm{C}$ for 24 hours. Then, samples were neutralised by $1 \mathrm{M} \mathrm{HCl}$ and then centrifuged for 5 minutes at $1000 \mathrm{rpm}$. The supernatants were analysed by PierceTM BCA Protein Assay Kit. This assay is based on the calorimetric method and used bicinchromic acid (BCA) to determine and quantify the total protein. This technique works by a combination of reduction of $\mathrm{Cu}+2$ to $\mathrm{Cu}+1$ using a protein in the alkaline medium with the highly selective and sensitive detection of $\mathrm{Cu}+1$ cation using a reagent contains bicinchoninic acid. This reaction leads to chelation of one cuprous ion with two molecules of BCA, which produces a purple color. By increasing the concentration from 20 to $2000 \mu \mathrm{g} / \mathrm{mL}$, a linear line can be generated when the absorbance is measured at $562 \mathrm{~nm}$ (Thermofisher.com, 2018). The absorbance of the combination of BSA NPs and working agents were measured using xMarkTM Microplate Absorbance Spectrophotometer (Bio-Rad, USA) at $562 \mathrm{~nm}$. A comparison between the absorbance of BSA NPs and the BSA standard calibration in the range from 0 to $200 \mu \mathrm{g} / \mathrm{ml}$ provides us the actual bovine serum concentration. The encapsulation efficiency was calculated by the ratio between the actual bovine serum albumin and the theoretical serum albumin concentration, as described in the following equation:

Encapsulation efficiency $\%=$ (the actual bovine serum albumin concentration/ theoretical serum albumin concentration) $\times 100 \%$.

\subsection{In vitro release study of $\mathrm{BSA} \mathrm{NP}$.}

$5 \mathrm{mg}$ of BSA NPs were diffused in $1.0 \mathrm{ml}$ of phosphate buffered saline solution (PBS, PH 7.4). Samples were incubated at $37^{\circ} \mathrm{C}$ and then the release medium was taken at intervals 24 hours and 168 hours and replaced with fresh medium at each time. The supernatant was analysed using PierceTM BCA Protein Assay Kit and then measured using xMarkTM Microplate Absorbance Spectrophotometer (Bio-Rad, USA) at $562 \mathrm{~nm}$. A comparison between the absorbance of BSA NPs and the BSA standard calibration in the range from 0 to $200 \mu \mathrm{g} / \mathrm{ml}$ provides us with the actual bovine serum concentration.

\subsection{Determination of interactions between BSA, PLGA, PVA, PCL by FTIR.}

Fourier transform infrared spectrophotometer FTIR (IRAffinity-1S-SHIMADZU®) was applied to examine the chemical interactions between nanoparticles and polymers. BSA NPs, PCL, and PLGA interactions were examined using IRAffinity-1S, SHIMADZUß. The FTIR spectrum was analysed in the range from $500-4000 \mathrm{~cm}-1$. FTIR can detect the functional groups in the nanoparticles and raw materials. By comparing the nanoparticles spectrum and their row materials spectrum, FTIR spectrum aids us to compare between the nanoparticles and the row materials. The BSA NPs were treated prior to the loading in FTIR and then compared with the raw materials spectrums (BSA, PLGA, PCL) using Labsolutions IR software.

\section{Results and Discussion.}

\subsection{Scanning electron microscopy.}

The morphological study was performed on BSA NPs prepared either by double emulsion or microfluidic techniques. The study was held to compare the geometrical shapes and sizes of the formulated NPs. Figures (7- 10) displayed below, show BSA loaded PLGA and BSA 
loaded PCL NPs prepared by either the double emulsion method or the microfluidic technique. All the NPs were examined on magnification $7 \mathrm{X}$, where further specifications are mentioned in table (2)

As noticed from the microscopic images, all BSA NPs showed spherical shapes. Figures (7 and 9) showed BSA NPs prepared by the double emulsion method, where a nonuniformed size distribution was noticed along with bigger particles size compared to the NPs obtained by the microfluidic technique displayed in figures ( 8 and 10). BSA loaded PLGA NPs ( 7 and 8) showed no significant difference in the particles size compared to BSA loaded PCL NPs in both techniques ( 9 and 10).

Figures (7 and 9) resembel BSA NPs prepared by the double emulsion method, wheras figures (8 and 10) show BSA NPs prepared by the microfluidic technique. Those results give a clear indication that the BSA NPs obtained by the microfluidic technique had lower PDI and smaller particles size compared to those prepared by the double emulsion technique. On the other hand, no clear determination was possible in the case of BSA loaded PLGA and BSA loaded PCL NPs .A possible reason might be due to the slightly difference in the particles size between both polymers.

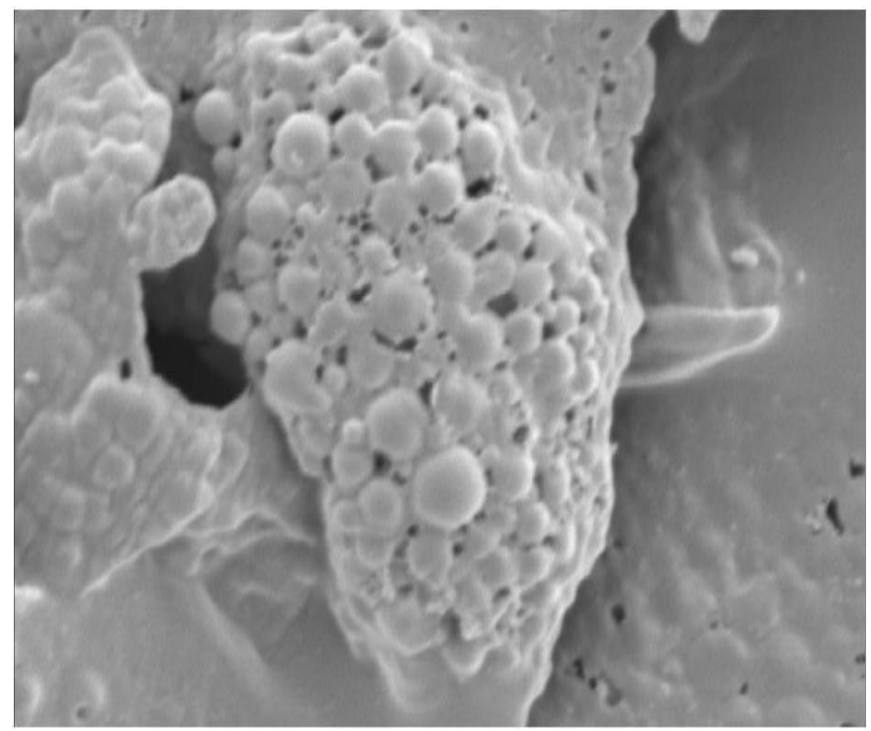

Figure (7). showing the image of SEM for BSA loaded PLGA prepared by double emulsion technique.

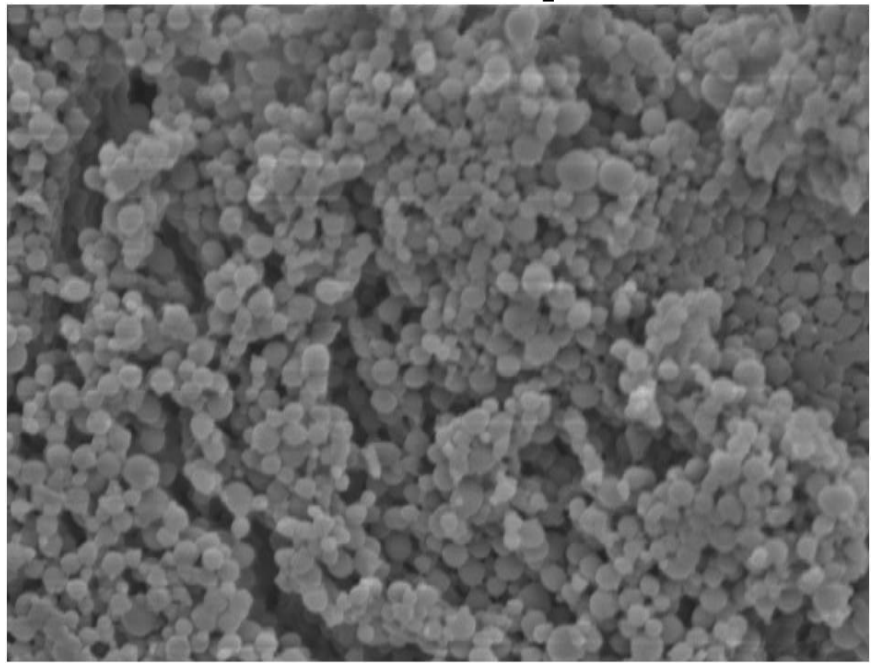

Figure (8). showing the image of SEM for BSA loaded PLGA prepared by microfluidic technique. 


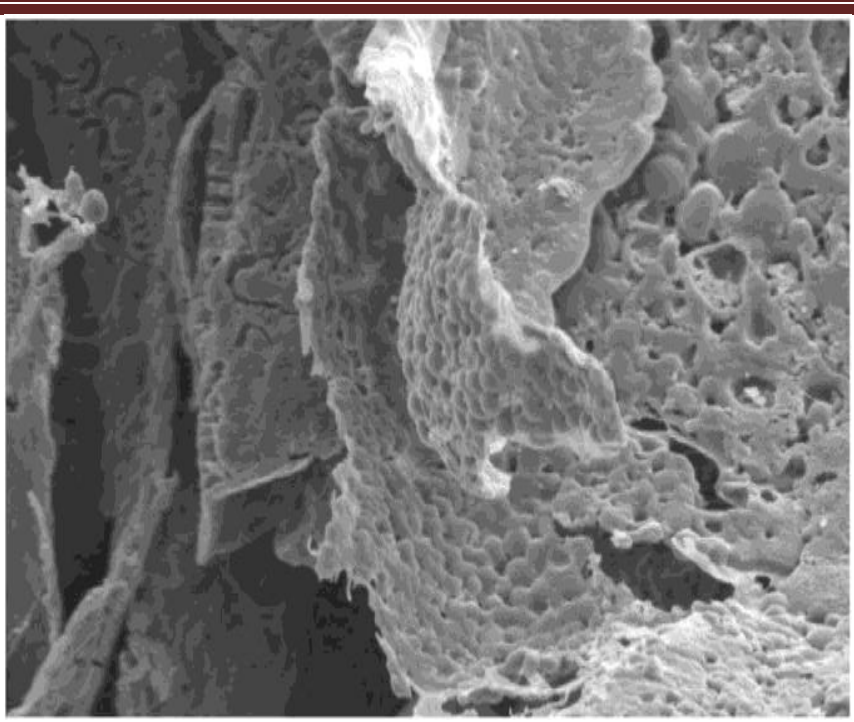

Figure (9). showing the image of SEM for BSA loaded PCL prepared by double emulsion technique.

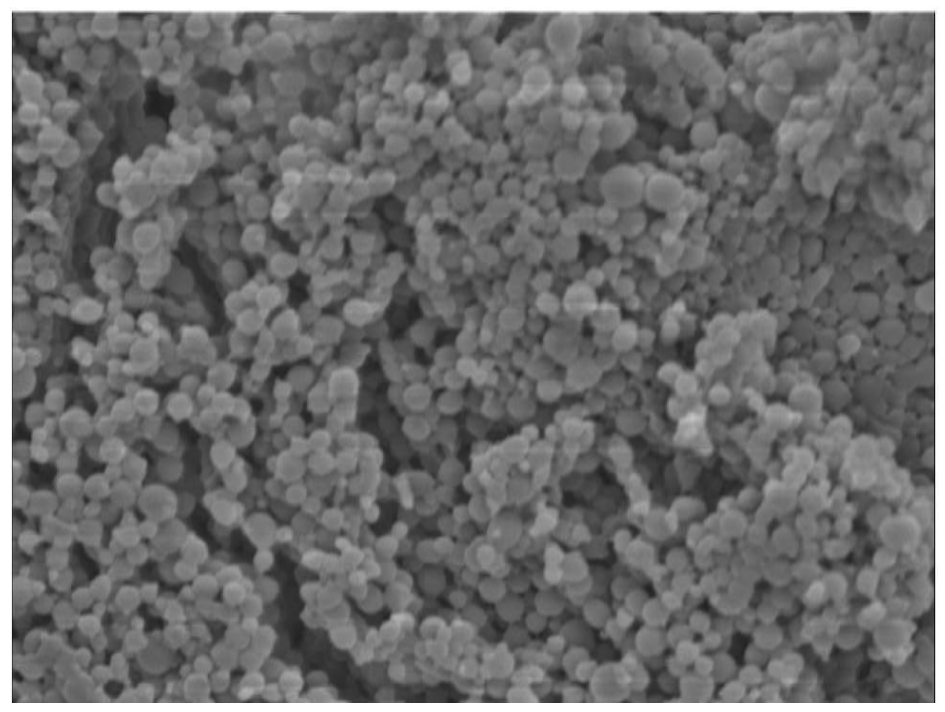

Figure (10): showing the image of SEM for BSA loaded PCL prepared by microfluidic technique.

Table (2): Showing the details about the images of SEM for BSA NP prepared by double emulsion and microfluidic techniques

\begin{tabular}{|l|l|l|l|}
\hline & DATASIZE & Magnification & Working distance \\
\hline PLGA DE & $1280 \times 960$ & 7000 & $17.9 \mathrm{~mm}$ \\
\hline PCL DE & $1280 \times 960$ & 7000 & $17.7 \mathrm{~mm}$ \\
\hline PLGA MF & $1280 \times 960$ & 7000 & $5.1 \mathrm{~mm}$ \\
\hline PCL MF & $1280 \times 960$ & 7000 & $5.0 \mathrm{~mm}$ \\
\hline
\end{tabular}


4.2. Particle size distribution (PSD) and Polydispersity index (PDI).

4.2.1. Particles size distribution (PSD).

The particles size distribution is a fundamental parameter, which affect the properties of nanoparticles in several ways such as surface area, permeability and eventually bioavailability. The measurement of the particles size distribution is a critical key to explain the size of nanoparticles. The particles size distribution of polymers prepared using the double emulsion technique shown in figure (11). In PLGA NPs, the particles size distribution assigned with $528.1 \mathrm{~nm}$ showed more precipitate formation and aggregation of the nanoparticles. While, the particles size distribution designated with $528.1 \mathrm{~nm}$ showed less precipitate formulation and aggregation of the nanopartilces. Additionally, Abdelkader and his colleagues showed that the particles size distribution with $215 \mathrm{~nm}$ showed less aggregation with no precipitate formation, which was suggested to be the optimum particles size average of PLGA (Abdelkader et al., 2018). Therefore, Abdelkader study supports the results obtained from this study, where the particles size average is relatively the same. In the PCL NPs, the particles size distribution with $812 \mathrm{~nm}$ showed also more precipitate formulation and aggregation, as seen in figure (11). Whereas, the particles size average with $260 \mathrm{~nm}$ showed less precipitate formulation and aggregation. Therefore, a study done by Lamprecht showed particles size distribution with $260 \mathrm{~nm}$, which matches the results obtained from this investigation (Lamprecht et al., 2000). Yet, Lamprecht study does not support the average size of $812 \mathrm{~nm}$ obtained. This shows that the $812 \mathrm{~nm}$ is not considered as a suitable NPs average size. However, all the results from the double emulsion technique displayed particles size distribution in the nanoparticles range (less than $1 \mu \mathrm{m}$ ).

The particles size distribution of formulations prepared by microfluidic technique shown in the figure (12). Unlike double emulsion, all the mean particles size had an optimum size between $200 \mathrm{~nm}-260 \mathrm{~nm}$. In the PLGA NPs prepared by microfluidic technique, the particles sizedistribution was slightly smaller compared to those prepared by the double emulsion technique. Also, in the PCL NPs prepared by the microfluidic technique, the particles size distribution was slightly smaller compared to those prepared by the double emulsion technique. Those results show that BSA NPs prepared by the microfluidic technique had smaller particles size distribution than those prepared by the double emulsion technique.

\subsubsection{Polydispersity index (PDI).}

Poly dispersity index (PDI) is the average of non-uniformity nanoparticles where the range from 0-1. The higher PDI value means more non-uniform nanoparticles (polydispersity). The lower PDI value means less non-uniformity of nanoparticles (monodispersity). In this study, all polymeric nanoparticles formulations prepared either by the double emulsion or microfluidic technique had polydipersity range from 0.105 to 0.553 , as seen in figures (11 and 12).

In this present study, the PDI of BSA NP prepared by double emulsion technique was 0.162 , $0.315,0.211$, and 0.166 for PLGA NPs, as seen in figure (11). The PDI of BSA NPs prepared by microfluidic technique was $0.134,0.122,0.110$, and 0.129 for PCL NPs, as seen in figure (12). All polymer nanoparticle formulations prepared by double emulsion had significantly higher PDI compared to those nanoparticles prepared by microfluidic technique, as seen in table (3) comparing the obtained results. Those results mean that BSA NPs prepared by the microfluidic technique had more monodispersed nanoparticles or more homogeneity than BSA NPs prepared by double emulsion technique. Therefore, all nanoparticles prepared by microfluidic technique had the lowest PDI in around 0.100 range. 

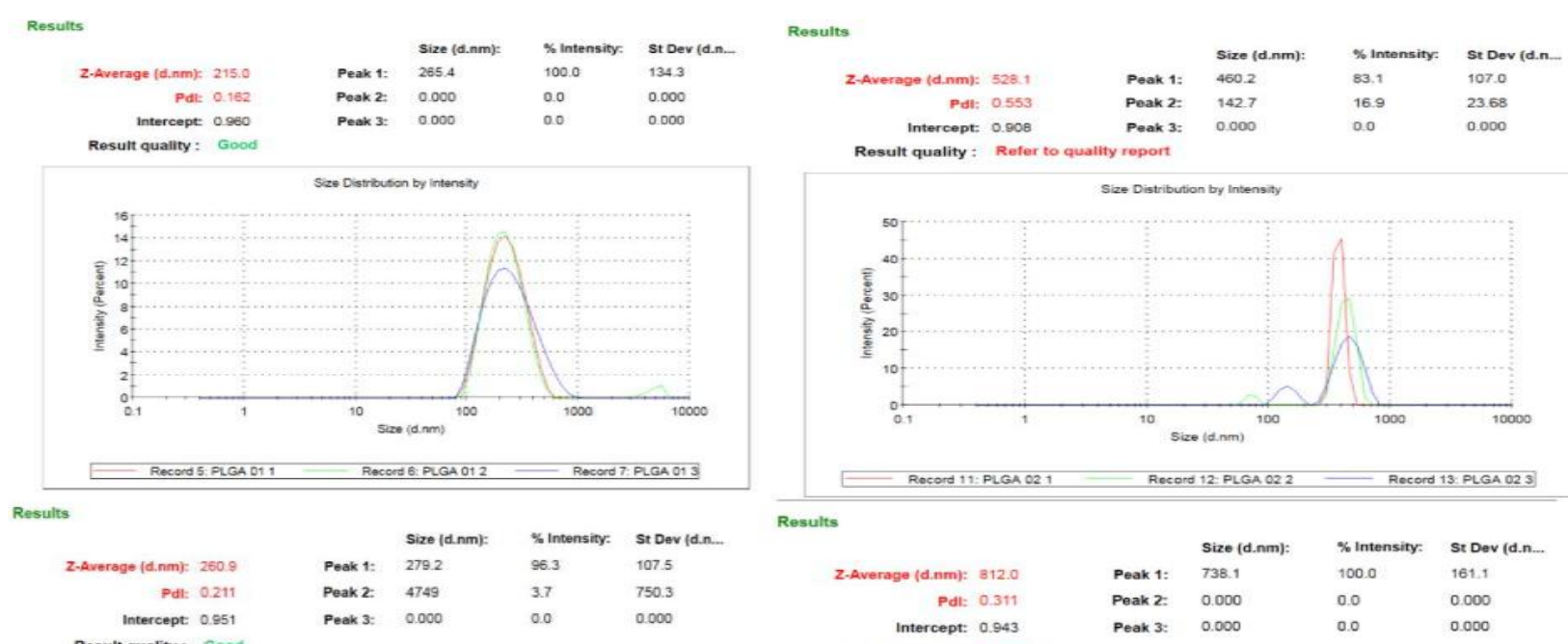

Result quality: Good

Size Distribution by intensity

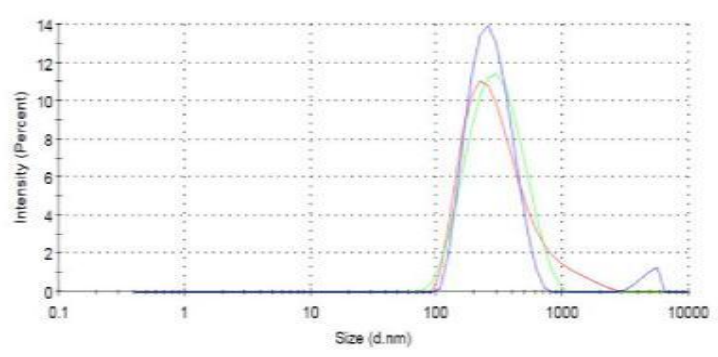
Result quality : Refer to quality repor
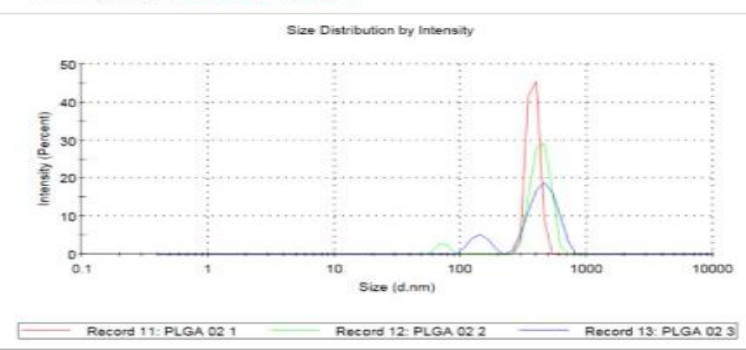
Results

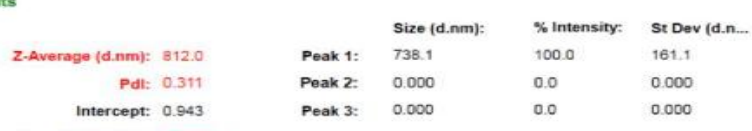

Result quality: Good

Record 23: PCL $021 \div$ Record 24: PCL $022-$ Recoed 25: PCL 023

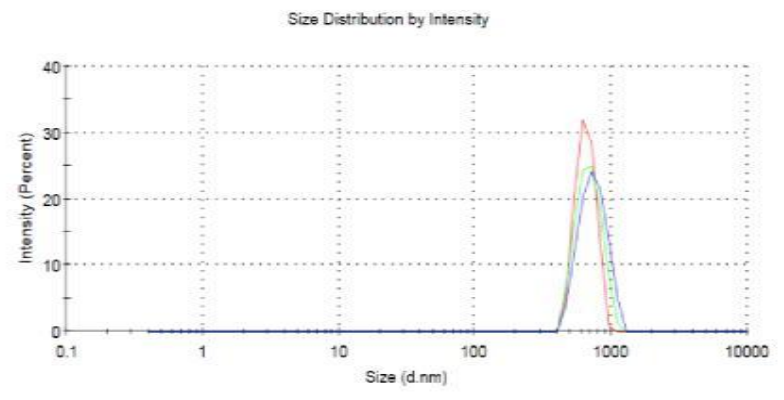

Figure (11): Showing the particles size distribution and PDI of PLGA NP and PCL NP prepared by double emulsion technique
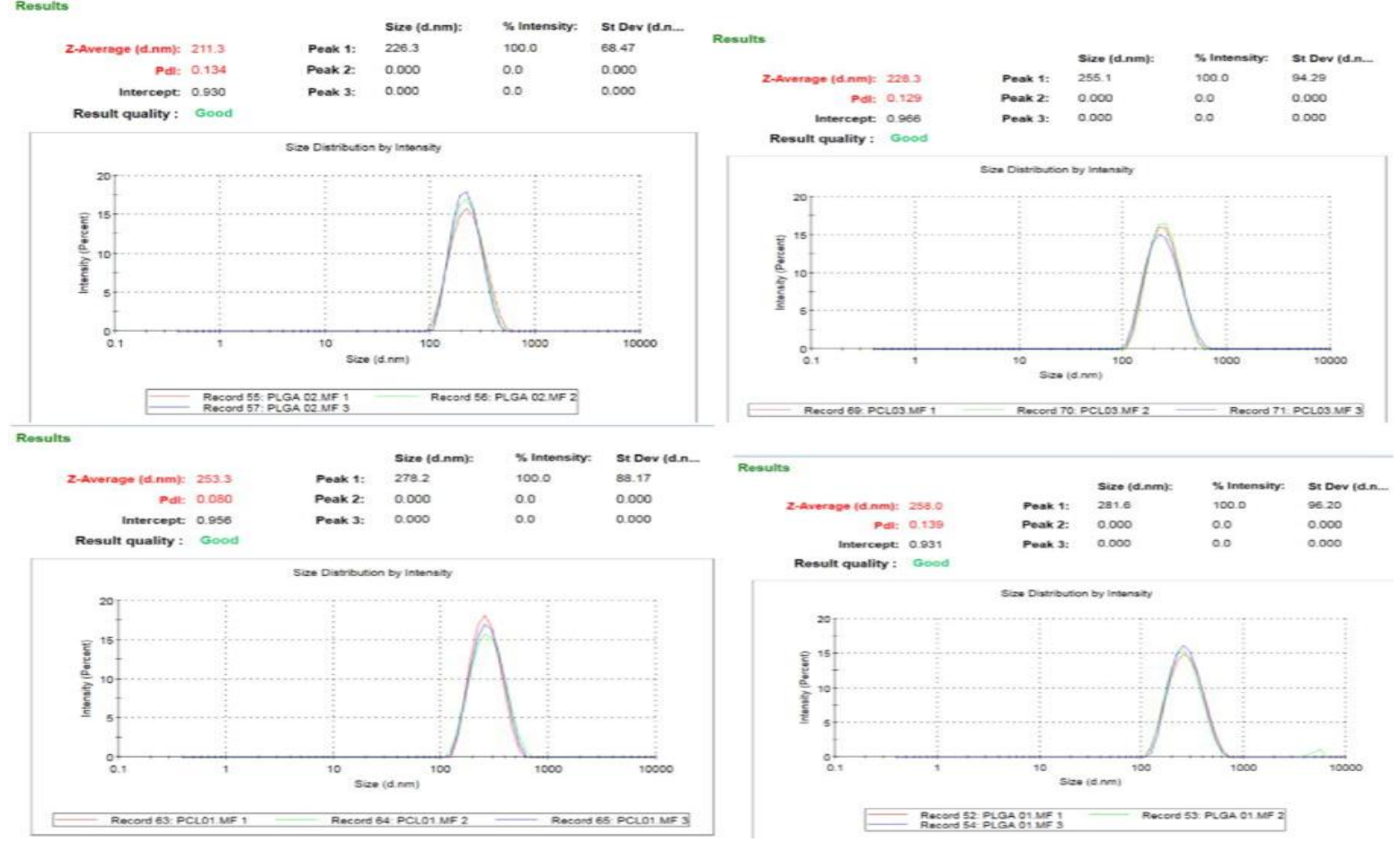

Figure (12): Showing the particles size distribution and PDI of PLGA NP and PCL NP prepared by microfluidic technique. 
Table (3): showing the size, and PDI of BSA NP obtained from double emulsion and microfluidic techniques.

\begin{tabular}{|c|c|c|c|c|c|}
\hline Formulation & Technique & $\begin{array}{l}\text { Siz } \\
\text { e } \\
\text { nm }\end{array}$ & $\begin{array}{l}\text { size } \mathbf{~ n m} \\
(\text { mean) }\end{array}$ & PDI & $\begin{array}{l}\text { PDI } \\
\text { average }\end{array}$ \\
\hline PLGA 01 & \multirow{6}{*}{$\begin{array}{l}\text { Double emulsion technique } \\
\text { solvent evaporation }\end{array}$} & 215 & \multirow{3}{*}{400.766} & 0.375 & \multirow{3}{*}{0.3633} \\
\hline PLGA 02 & & 528.1 & & 0.553 & \\
\hline PLGA 03 & & 459.2 & & 0.162 & \\
\hline PCL 01 & & 812 & \multirow{3}{*}{445.233} & 0.166 & \multirow{3}{*}{0.229} \\
\hline PCL 02 & & 260.9 & & 0.211 & \\
\hline PCL 03 & & 262.8 & & 0.311 & \\
\hline PLGA 01 & \multirow{6}{*}{ Microfluidic technique } & 253.3 & \multirow{3}{*}{231.133} & 0.08 & \multirow{3}{*}{0.112} \\
\hline PLGA 02 & & 211.3 & & 0.134 & \\
\hline PLGA 03 & & 228.8 & & 0.122 & \\
\hline PCL 01 & & 258 & \multirow{3}{*}{238.866} & 0.139 & \multirow{3}{*}{0.126} \\
\hline PCL 02 & & 230.3 & & 0.110 & \\
\hline PCL 03 & & 228.3 & & 0.129 & \\
\hline
\end{tabular}

The BSA NPs prepared by microfluidic technique had smaller mean particles size distribution compared to BSA NPs prepared by double emulsion technique, as seen in figure (13). The reasons for that, in the double emulsion technique, BSA NPs required high she aring and homogenisation during single and double emulsion. Therefore, nanoparticles size are sensitive to several process parameters of double emulsion technique such as homogenised time and high or low shear. In this study, the nanoparticles size with broad size distribution had not enough homogenisation time, as seen in $528.1 \mathrm{~nm}, 459.5 \mathrm{~nm}$, and $812 \mathrm{~nm}$ for PLGA NPs. Also, the double emulsiuon technique had multipile emulsification steps, which leads to a larger distribution of the nanoparticles, as seen in figure (14). Moreover, the homogenisation process could affect BSA stability. The higher shearing elevates the temperature of nanoparticles leading to protein denature, which eventually gives more emulsion coalescence with high mean particle size distribution (Zambaux, 1998).

In contrast, the microfluidic technique requires one step to form percise drops without the necessity of multiple emulsifications as in the double emulsion method, thus providing optimum drop size by microchannels present in both continous phase and dispersed phase. 
Further, the microfluidic technique generates nanoparticles in only one step without homogenisation process, which leads to smaller particles size with low PDI, as seen in figures (13 and 14). Also, because of the microcapillaries present in the nanoassembler cartridge, the microfluidic technique controls over the number and size of the inner droplets (Chu et al., 2007). This means microfluidic technique is able to produce highly monodispersed particles ,due to the microcapillaries, which provide high pressure to the nanoparticles. Therefore, the BSA NPs prepared by double emulsion had comparatively heterogeneous particles size compared to the BSA NPs prepared by the microfluidic technique.

PLGA NPs had slightly smaller particles size distribution than PCL NPs in both techniques, as seen in figure (13). The molecular weight of the polymer affects the particles size distribution. An increase of the molecular weight of the polymer will increase the particles size distribution by increasing the monomer components (Dinarvand et al., 2011). PLGA polymer had slightly lower molecular weight compared to PCL polymer. Thus, the particles size distribution with BSA PLGA $(400 \mathrm{~nm}$ and $231 \mathrm{~nm})$ were slightly smaller in size compared to PCL NPs (445nm and $235 \mathrm{~nm}$ ) in both techniques, as seen in the figure (13). Moreover, the physicochemical properties of polymers play an important role in the determination of the particles size distribution such as the solubility of the polymer in organic solvent, crystallinity, melting point, hydrophobicity, and viscosity. PLGA polymer is more hydrophilic than PCL polymer, due to the presence of PGA in PLGA structure and PLA in PCL structure (Schliecker et al., 2003). Therefore, PLGA polymer is favorable to interact along with BSA and thus reducing the particles size distribution compared to the PCL polymer, as seen in figure (13).

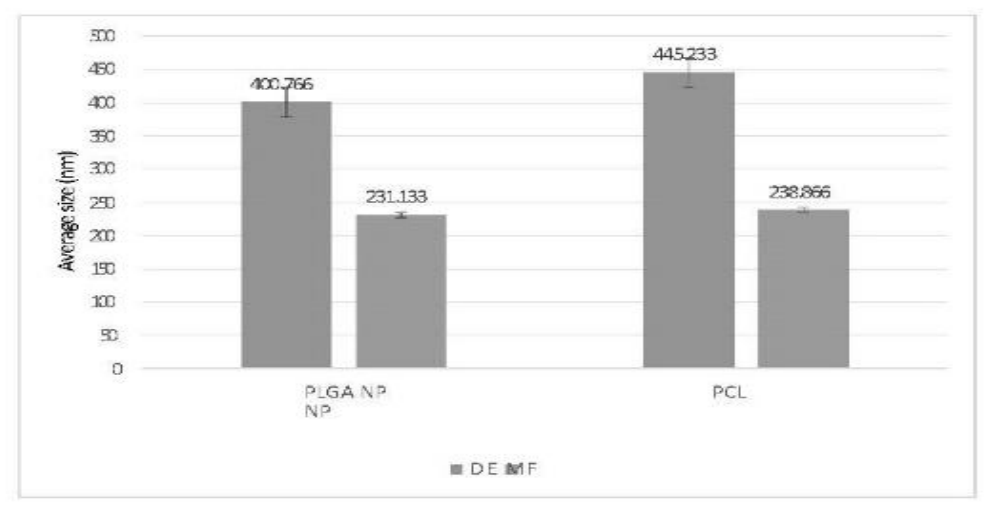

Figure(13): showing the average of size of BSA NP prepared by double emulsion and microfluidic technigues.

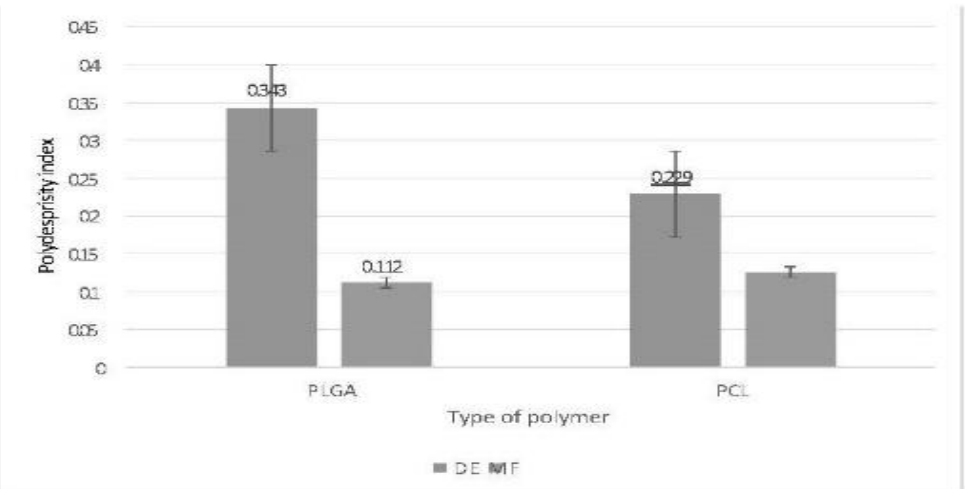

Figure (14): showing the average of PDI of BSA NP prepared by double emulson and microfluidic techniques. 


\subsection{Zeta potential.}

Zeta potential is a technique that used to determine the surface charge of nanoparticles in solution (colloid) and one of the major parameters known to affect stability of nanoparticles. Therefore, both the PLGA NPs prepared by double emulsion and microfluidic techniques exhibited negative charge zeta potential values $-7.21 \mathrm{mV}$, and $-7.96 \mathrm{mV}$ respectively, as seen in table (4). The PCL nanoparticles prepared by double emulsion or microfluidic techniques exhibited negative charge zeta potential values $-7.96 \mathrm{mV}$ and $-7.46 \mathrm{mV}$ respectively as seen in table (4). The NPs obtained by neither the double emulsion nor the microfluidic techniques had significant effect on the zeta potential as seen in the table (4). Yet, this result was not expected since double emulsion and microfluidic techniques had different organic solvents; Dichloromethane and Acetonitrile respectively, which leads to affects the zeta potential values.

Table (4): showing the Zeta Potential of BSA NPs obtained from double emulsion and microfluidic techniques.

\begin{tabular}{|c|c|c|c|}
\hline Formulation & Technique & $\begin{array}{l}\text { Zetasize } \\
(\mathrm{mV})\end{array}$ & $\begin{array}{l}\text { Zeta Potential (mV) } \\
\text { average }\end{array}$ \\
\hline PLGA 01 & \multirow{6}{*}{$\begin{array}{l}\text { Double emulsion technique } \\
\text { / solvent evaporation }\end{array}$} & -9.18 & \multirow{3}{*}{-7.21} \\
\hline PLGA O2 & & -5.13 & \\
\hline PLGA 03 & & -7.34 & \\
\hline PCLO1 & & -5.6 & \multirow{3}{*}{-7.96} \\
\hline PCL O2 & & -8.3 & \\
\hline PCi. 03 & & -10 & \\
\hline PICAA OI & \multirow{6}{*}{ Microfluidie lechnique } & -7.2 & \multirow{3}{*}{-7.46} \\
\hline PLGA 02 & & -8.5 & \\
\hline PLGA 03 & & -6.7 & \\
\hline PCLOI & & -7.9 & \multirow{3}{*}{-7.96} \\
\hline PCL 02 & & -5.9 & \\
\hline PCL 03 & & -10.1 & \\
\hline
\end{tabular}

\subsection{Encapsulation efficiency and loading of BSA.}

The encapsulation efficiency is an important parameter, which detects how much drug has been encapsulated by either double emulsion or microfluidic technique. Each formulation weight was measured and then the average was calculated. The encapsulation efficiency was determined by soaking the BSA NPs into $1 \mathrm{M} \mathrm{NaOH}$, then incubating and examining the released BSA NPs with microplate reader at $562 \mathrm{~nm}$ after applying PierceTM BCA Protein Assay. PierceTM BCA Protein Assay is by a comparison between proteins (BSA) samples and proteins standard, which gives the unknown concentration of samples via spectrophotometer. 
The concentrations of PLGA and PCL NP obtained by double emulsion technique were calculated by using the equation of standard curve of BSA $y=0.0059 x+0.0598$, as seen in the figure (15). Therefore, the average of encapsulation efficiency for PLGA and PCL prepared by double emulsion technique calculated by ratio between the actual bovine serum albumin and theoretical serum albumin concentration, as seen in the table (5). In the microfluidic technique, the encapsulation efficiency was measured by the same method. The equation of standard BCA assay was $\mathrm{y}=0.0095 \mathrm{x}+0.1184$, as seen in this figure (16). Therefore, the average encapsulation efficiency of PLGA and PCL prepared by microfluidic displayed in the table (6).

As shown in figure (17), the encapsulation efficiency of BSA loaded PLGA prepared by double emulsion technique was $27 \%$ showing higher encapsulation capability that that of BSA loaded PCL prepared by double emulsion technique, which was $23.3 \%$. As for the microfluidic technique, BSA loaded PLGA showed an encapsulation efficiency of $60.45 \%$ compared to the BSA loaded PCL 42\%. Therefore, it is concluded that the entrapment efficiency in PLGA NPs was higher than PCL NPs in both techniques.

Table (5): showing the encapsulation efficiency of PLGA and PCL prepared by double emulsion technique in BCA Protein Micro Assay Kit

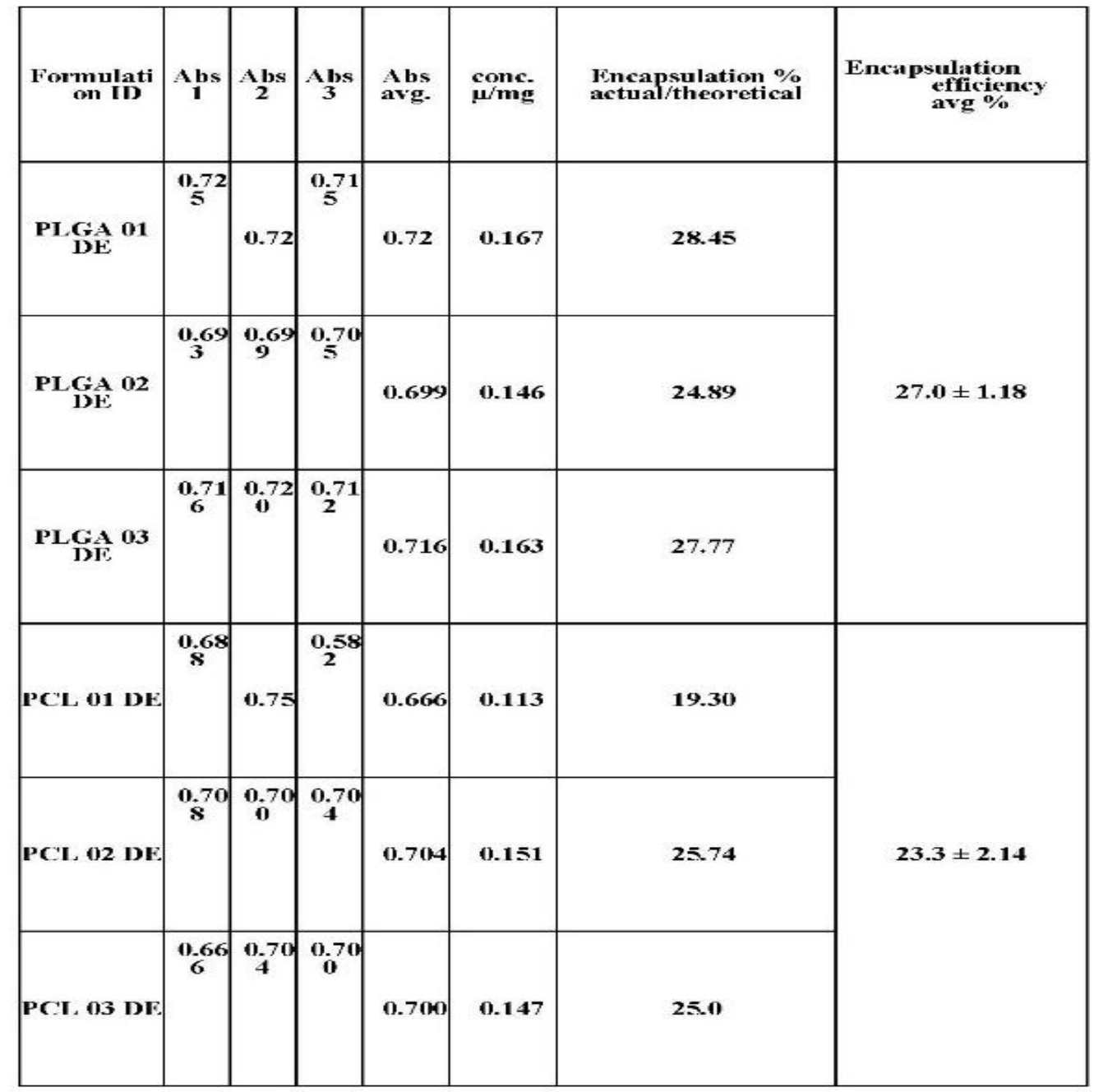




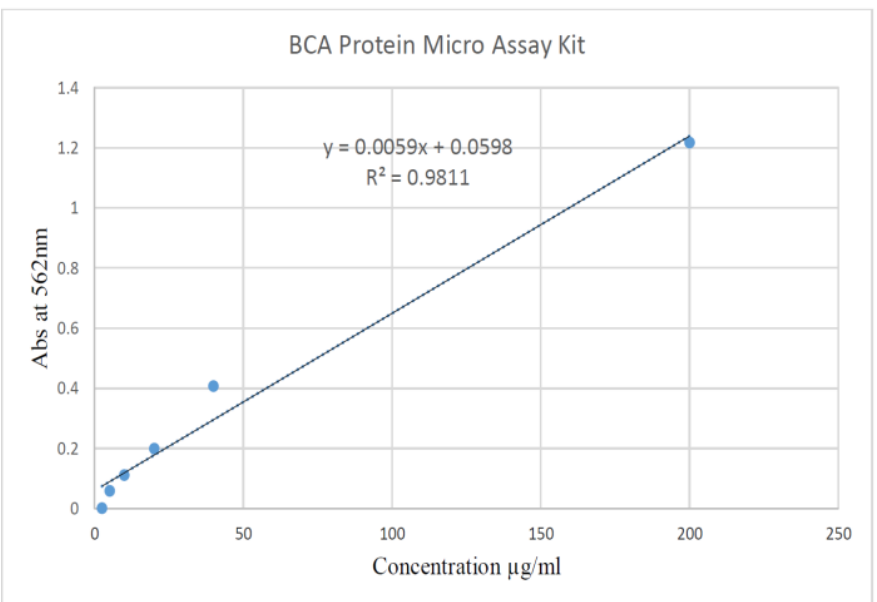

Figure (15): Showing the Standard curve of BCA Protein Micro Assay Kit.

Table (6): showing the encapsulation efficiency of PLGA and PCL prepared by microfluidic technique in BCA Protein Micro Assay Kit

\begin{tabular}{|c|c|c|c|c|c|c|c|}
\hline$\underset{\text { ID }}{\text { Formulation }}$ & $\underset{1}{\mathrm{Abs}}$ & $\underset{2}{\mathrm{Albs}}$ & $\underset{3}{\Delta b s}$ & $\begin{array}{l}\text { Abs } \\
\text { avg. }\end{array}$ & $\begin{array}{l}\text { conc. } \\
\mu / \mathrm{mg}\end{array}$ & $\begin{array}{l}\text { Encapsulation \% } \\
\text { actual/theoretical }\end{array}$ & $\begin{array}{l}\text { Encapsulation } \\
\text { cfficiency avg } \\
\%\end{array}$ \\
\hline PIGA 01 DF & $\begin{array}{c}0.80 \\
0\end{array}$ & 0.85 & 0.89 & 0.855 & 0.691 & 72.8 & \\
\hline PLGA 02 DE & 0.60 & 0.65 & 0.70 & 0.65 & 0.486 & 51.22 & $60.45 \pm 7.19$ \\
\hline PLGA O3 DE & \begin{tabular}{c|}
0.71 \\
6
\end{tabular} & 0.72 & $\begin{array}{c}0.71 \\
2\end{array}$ & 0.71 & 0.546 & 57.34 & \\
\hline PCL 01 DE & \begin{tabular}{c|}
0.66 \\
0
\end{tabular} & $\frac{0.65}{5}$ & $\begin{array}{c}0.65 \\
0\end{array}$ & 0.655 & 0.49 & 51.74 & \\
\hline PCI, 02 DF & 0.45 & 0.44 & $\begin{array}{c}0.45 \\
1\end{array}$ & 0.451 & 0.284 & 30.27 & $42.0 \pm 7.15$ \\
\hline PCL 03 DE & $\frac{0.58}{2}$ & 0.57 & $\begin{array}{c}0.59 \\
0\end{array}$ & 0.582 & 0.41 & 44.0 & \\
\hline
\end{tabular}




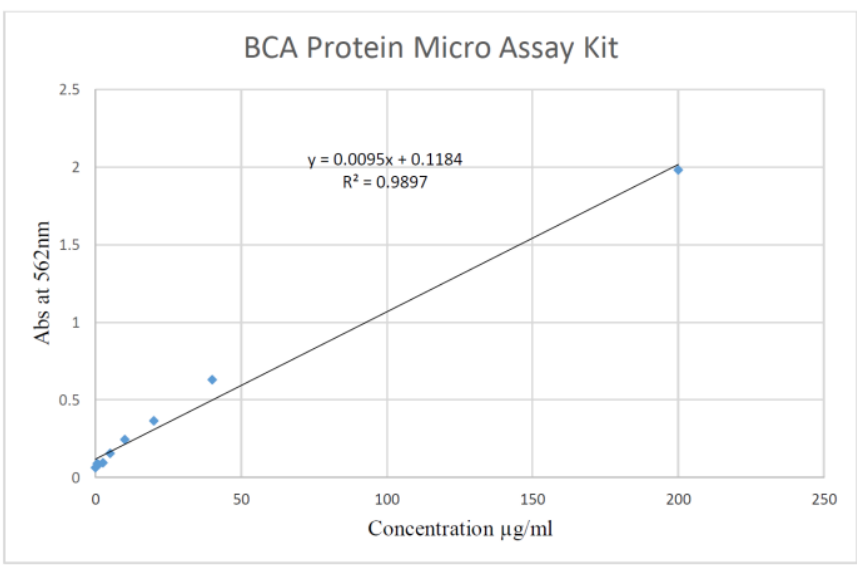

Figure (16): Showing the Standard curve of BCA Protein Micro Assay Kit.

Although the double emulsion technique is suitable for incorporated the hydrophilic drugs such as BSA, insulin, according to (Iqbal et al., 2018). Yet, this study showed that BSA NPs prepared by double emulsion seem to be unsuitable for BSA incorporation. The reasons might be related to BSA capability to escape from the internal aqueous phase to the external aqueous phase during the single emulsion or may not become dissolved completely in the organic solvent in the double emulsion preparation leading to lower entrapment. On the other hand, since the microfluidic technique composed of one step, BSA would have lower chances to escape to the external environment. Moreover, the drops obtained by microfluidic technique allow producing bubbles around the produced drops, thus providing extremely high encapsulation efficiency with higher stability (Wang, Zhang and Chu, 2014).

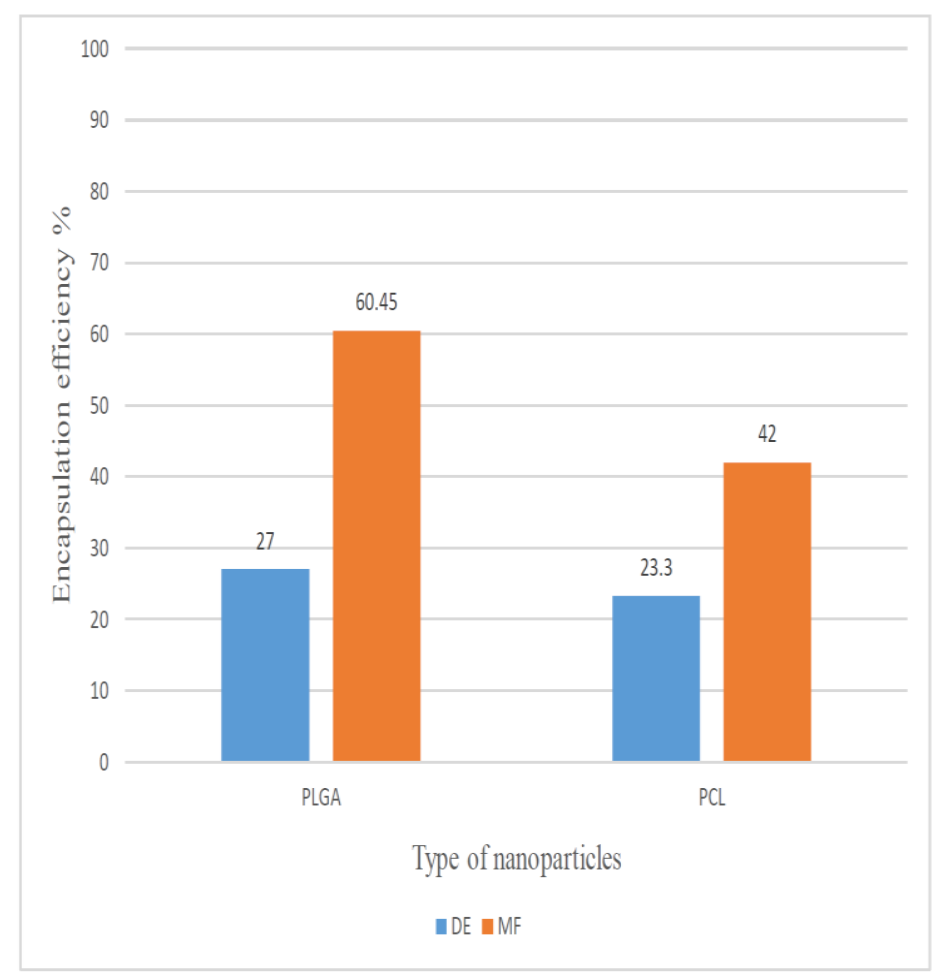

Figure (17): showing the encapsulation efficiency of BSA NP prepared by double emulsion technique and microfluidics techniques 
In addition, according to ( $\mathrm{Li}, 1999)$, the protein encapsulation efficiency depends on the thickness of the organic phase compared to the internal aqueous solution. Therefore, an increase in the volume of the external phase might lead the drug to leave towards the external aqueous phase. In this study, the organic phase was used in both techniques. The volume utilized in the double emulsion was $10 \mathrm{ml}$ of DCM, while the organic solvent used in microfluidic technique was only $4 \mathrm{ml}$ of acetonitrile. Therefore, the double emulsion technique had thickener organic phase compared to the microfluidic technique, which as expected, showed lower encapsulation efficiency in comparison to the microfluidic technique as seen in figure (17).

Moreover, encapsulation of protein in nanoparticles prepared by double emulsion technique used either sonication or homogenization, which could lead to unfolding or aggregation of the BSA. In other words, high rates of shears lead to instability of BSA, thus resulting in low encapsulation efficiency of nanoparticles. In microfluidic technique, the technique did not implement any sonication or homogenisation. Therefore, the microfluidic method is potentially favoured to encapsulate BSA with high amounts. This study results suggest that microfluidic technique has a privilege for the preparation of polymer nanoparticles over the double emulsion technique.

Despite having both polymers slightly difference in particle size average, the encapsulation efficiency of nanoparticles was observed a massive difference between two polymers. The physicochemical properties of polymer play a fundamental rule for determining the encapsulation efficiency. In this study, the encapsulation efficiency study was observed that the BSA NP made from PLGA polymer had higher encapsulation efficiency than those nanoparticles made from PCL polymer in both techniques, as seen in figure (17). Those results directly related to several parameters such as physicochemical properties of polymers and drug-polymer interaction and solubility the polymers in organic phase.

Firstly, the molecular weight of polymers effects on encapsulation efficiency according to this study (Dinarvand et al., 2011). As mentioned before, PLGA polymer has lower molecular weight than PCL polymer. The low molecular weight gives more surface area available, which leads to increase the encapsulation efficiency.

In addition, the chemical compositions of polymer show an effect on the encapsulation efficiency. PLGA has more hydrophilic chains than PCL polymer. In this way, BSA is more favoured to encapsulate with PLGA polymers than PCL polymers in order to a better stabilizer at the internal phase. In other words, BSA diffuses to the external phase due to its instability with PCL polymers, thus decreasing the encapsulation efficiency (Martinez et al., 2017).

Moreover, the encapsulation efficiency depends on the particles size according to this study (Pessi et al., 2014). The particles size of PLGA were slightly smaller size than the particles size of PCL, thereby the encapsulation efficiency with PLGA polymer was higher than the encapsulation efficiency with PCL.

According to this work (Yeo and Park, 2004). Yeo and Park mentioned that the low solubility of polymers in organic solvent gives high encapsulation efficiency. Therefore, the solubility of PLGA in organic solvent is lower than the solubility of PCL in an organic solvent in both techniques, which used dichloromethane or acetonitrile as organic solvents. The low solubility in an organic solvent will solidify quicker, which allows to higher encapsulation efficiency. Therefore, as expected, the nanoparticles made from PLGA had higher encapsulation efficiency than nanoparticles made from PCL in both techniques, as seen in figure (17). 


\subsection{Release study.}

Drug release study is an important examination to investigate and establish the drug stability and therapeutics state of polymer nanoparticles. The drug release is the amount of drug that released from inside the nanoparticles into outside medium over the time. Drug release behaviour was performed by soaking the polymer nanoparticles into PBS and incubated at $37^{\circ} \mathrm{C}$ to achieve the environment of the human body. The release study was calculated by Micro BCA protein assay kit and measured using microplate reader at $562 \mathrm{~nm}$ on time intervals 24 hours and 168 hours, as shown in table (7). The figure (18) shows an initial burst release within the first 24 hours followed by a sustained release profile within 168 hours. An initial burst release and sustained release profile were observed in both techniques with both polymers as shown in figure (18). The initial release of BSA loaded PLGA prepared by double emulsion technique was $43.3 \%$ and the sustained release profile was $50 \%$. The initial release of BSA loaded PCL prepared by double emulsion technique was $30.86 \%$ and the sustained release was $69 \%$. The initial release BSA loaded PLGA prepared by microfluidic technique was $45.1 \%$ and the sustained release profile was $55 \%$. The initial release of BSA loaded PCL prepared by microfluidic technique was $43.8 \%$ and the sustained release profile was $50 \%$. BSA NP prepared by double emulsion technique had the less initial burst release and longer sustained release profile compared to those BSA NP prepared by microfluidic technique. In addition, BSA loaded PLGA had higher initial burst with shorter sustained release than BSA loaded PCL in both techniques, as seen in figure (18).

Table (7): showing the release study of the BSA loaded PLGA and BSA loaded PCL prepared by double emulsion and microfluidic techniques.

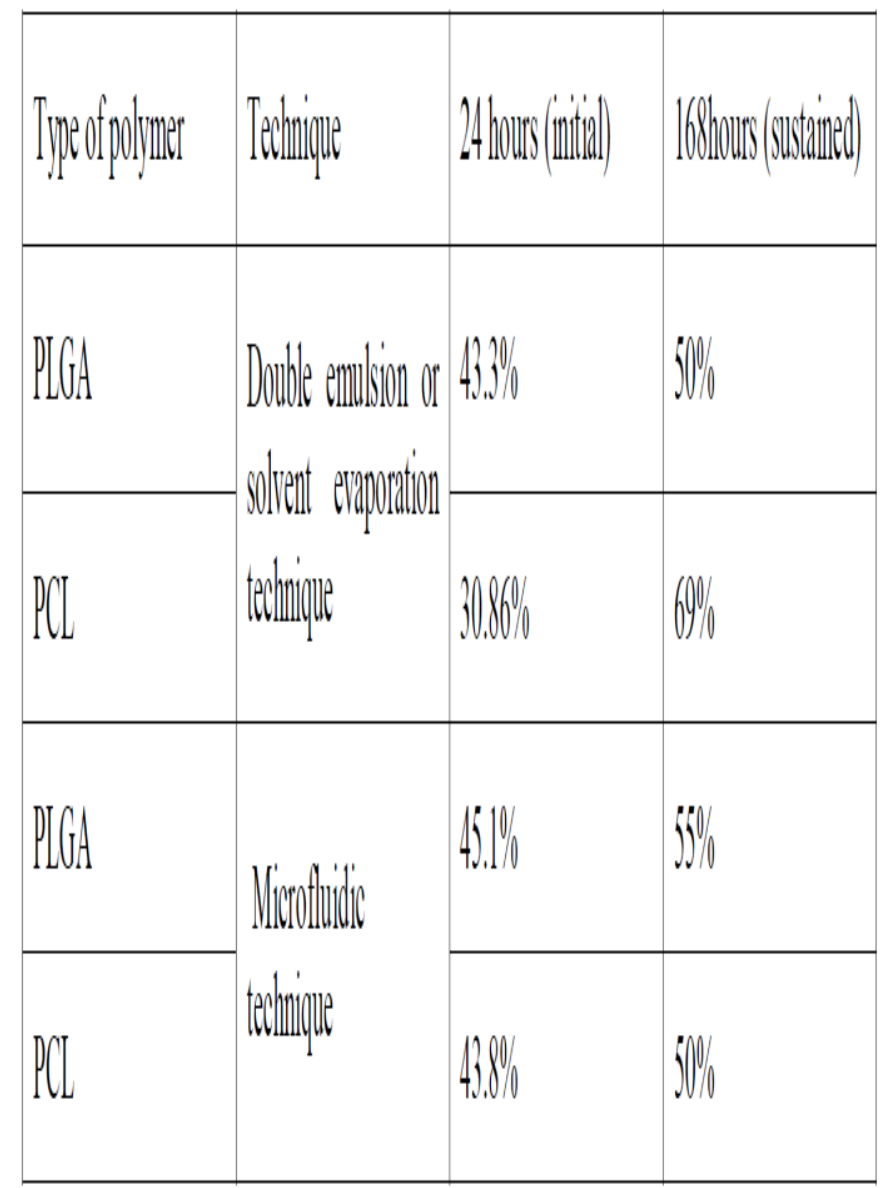




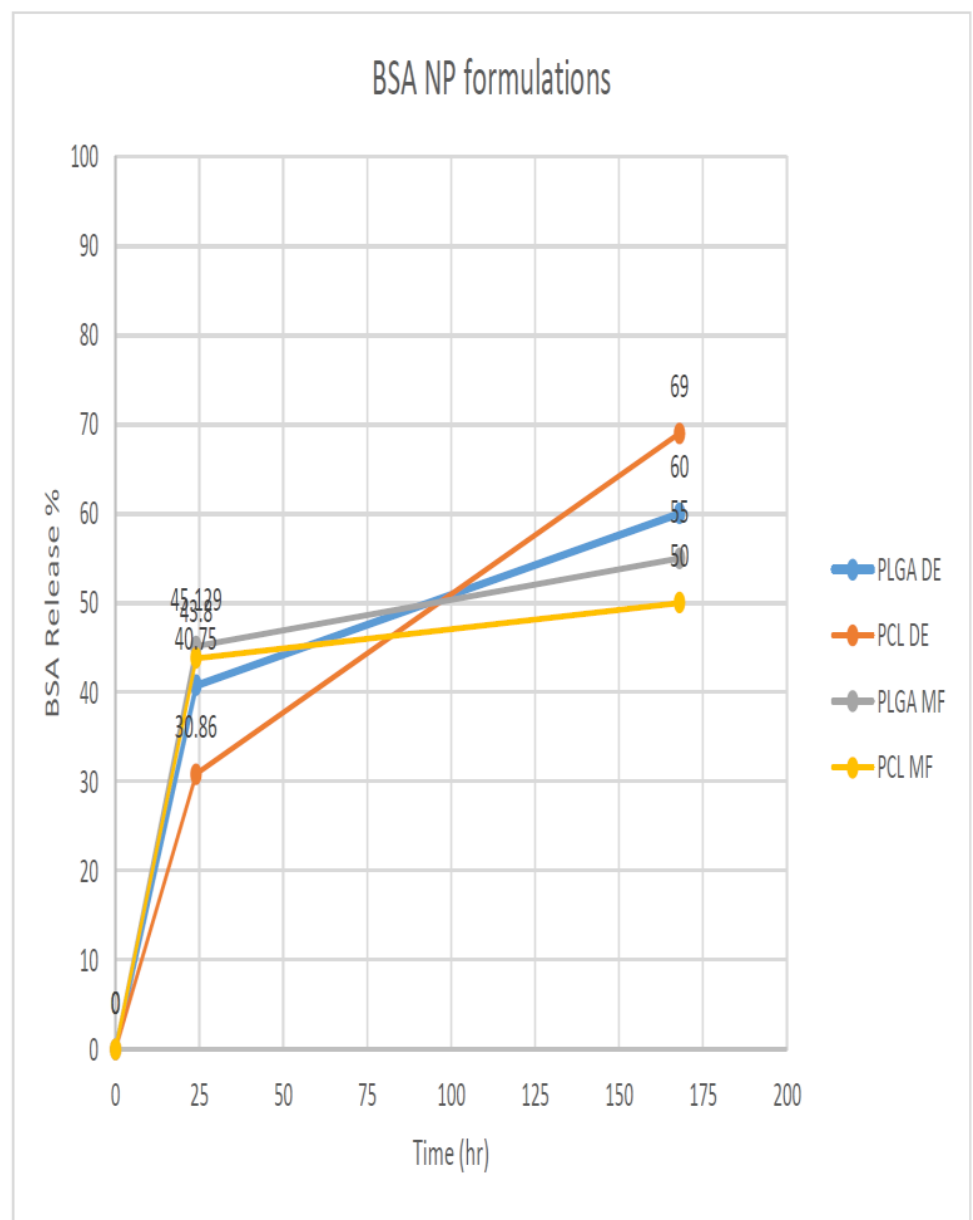

\section{Figure (18): showing the BSA release \% from PLGA DE, PCL DE, PLGA MF, and PCL MF.}

A comparison between BSA NP prepared by double emulsion and BSA NP prepared by microfluidic in the vitro release showed in figure (18). The initial drug release is related to the morphology of particles, the particles with more spherical form lead to increase the surface area available for drug release. Microfluidic technique provides an optimum spherical particle compared to double emulsion technique (Pessi et al., 2014). As expected, the BSA NP prepared by microfluidic technique had higher initial release than those prepared by double emulsion technique. As seen in figure (18), the grey and yellow lines were greater at 24 hours than the blue and orange lines.

In addition, encapsulation efficiency plays a fundamental rule to effect on an initial burst release. A higher encapsulation efficiency produces a higher initial burst, which leads to a faster release rate according to this study (Hans and Lowman, 2002). Therefore, as described before, the encapsulation efficiency of BSA NP prepared by microfluidic was higher than BSA NP prepared by double emulsion technique.

Moreover, BSA NP prepared by double emulsion had bigger external phase than BSA NP prepared by microfluidic. Therefore, the BSA NP prepared by double emulsion technique takes more time to release outside polymeric matrix than BSA NP prepared by microfluidic technique, which eventually leads to longer sustained release profile. As seen in figure (14), the blue and orange lines were greater at 168 hours than the grey and yellow lines. Also, the degradation of bovine serum albumin could lead to reduce the encapsulation efficiency during nanoparticles preparation. Homogenization of primary and secondary emulsion during 
NP fabrication allows producing the three dimensional in BSA structure. The instability effects on the vitro release study in double emulsion technique. On the other hand, the stability in the microfluidic technique is guaranteed during nanoparticles preparation in terms of not using homogenisers. The results suggest that BSA NP prepared by microfluidic technique had higher initial burst with shorter sustained release than BSA NP prepared by double emulsion technique, as seen in figure (18).

The different between PLGA and PCL polymers in burst release and sustained release showed in figure (18). The release polymer depends on parameters such as the molecular weight, Lactid/glycolide ratio, crystallinity and particle size.

Polymers with higher molecular weights contain a longer polymer chain, which demands more time to degrade, consequently resulting slower release rate (Allahyari and Mohit, 2015). PLGA has 24,000-35,000 molecular weight while PCL has 70,000-90,000 molecular weight, which means PLGA takes less time to degrade than PCL polymers. As expected, in both techniques, the initial release of BSA loaded PLGA was $(43.3 \%, 45 \%)$ greater than the initial release of BSA loaded PCL $(30.8 \%, 43.8)$, as seen in figure (18).

The lactide/glycolide ratio impacts on the hydrophilicity, crystallinity, and the hydration rate of the polymers. Polyglycolide acid (PGA) is more hydrophilic properties due to an absence of methyl side group compared to polylactide acid (PLA). Therefore, a polymer with a higher percentage of polyglycolide acid (PGA) is related to more water uptake, thereby resulting easily hydrated in an aqueous phase, which allows more water to penetrate into the BSA NP, thus providing to be released more (Park, 1995). PLGA showed more polyglycolide 50:50 (L/G) than PCL 85:15 (L/Caprolactone). Thus, the BSA loaded PLGA had the higher initial burst then BSA loaded PCL. Also, PCL polymer is characterised by a slow degradation due to its crystallinity and high olefinic properties.

In this study, the sustained release of BSA loaded PCL was $(69 \%, 50 \%)$ greater than the sustained release of BSA loaded PLGA $(50 \%, 55 \%)$ in both techniques, which as expected. Particles with large size have a less burst release with longer sustained release than particles with small size according to this study (Kumari, Yadav and Yadav, 2010). PLGA had smaller particles size than PCL particles size. In addition, the greater encapsulation efficiency gives a higher initial burst according to this study (Hans and Lowman, 2002). The results explained that BSA loaded PLGA nanoparticles had higher burst release with shorter sustained release than BSA loaded PCL nanoparticles in both techniques, as seen in figure (18).

\subsection{FTIR study.}

FTIR studies use to examine a possible interaction between BSA as drug model and the polymer nanoparticles as a drug carrier. FTIR spectrum was performance by determining the function groups of the BSA NP formulation and their raw materials. The FTIR spectra of raw materials and BSA NP formulations showed from $500 \mathrm{~cm}-1$ to $4000 \mathrm{~cm}-1$. All materials have been plotted and investigated in the FTIR spectra as shown in figures (19, 20, 21, and 22).

According to FTIR spectra, the function group of polymers showed at peak $1750 \mathrm{~cm}-1$ related to the carbonyl group $(\mathrm{C}=\mathrm{O})$, while the function group of PVA showed at peak 3300 $\mathrm{cm}-1$ related to the alcohol group $(\mathrm{O}-\mathrm{H})$ and the function group of pure BSA showed at peak $1680 \mathrm{~cm}-1$ related to primary amide group $(\mathrm{N}-\mathrm{C}=\mathrm{O})$.

In the figures $(19,20)$, the broad peak at $3300 \mathrm{~cm}-1$ is associated with $\mathrm{OH}$ group presence with PLGA NP and PCL NP, which means PVA has not removed successfully, because of the washing process during nanoparticles formulation. Importantly, all polymers had carbonyl group represent at $1750 \mathrm{~cm}-1$. Therefore, figures $(19,20,21$, and 22) displayed the matching between polymers and nanoparticles at $1750 \mathrm{~cm}-1$ on either double emulsion or microfluidic, while other peaks already disappeared. The results indicated BSA nanoparticles have been successful encapsulated. 
As seen in figures $(19,20,21$, and 22), all BSA NP formulations had no peak at $1680 \mathrm{~cm}-1$, because the amide group was not present in BSA NP. In other words, FTIR spectra of nanoparticles have not showed the BSA peak at $1680 \mathrm{~cm}-1$, which means that polymer nanoparticles encapsulated the BSA particles and provided more stability to BSA inside the nanoparticles. In the figures $(19,20,21$, and 22), the peaks of BSA NP formulations showed less sharp and more broad than polymers at $1750 \mathrm{~cm}-1$, which means the BSA NPs had fewer polymers concentration and found only polymers at the surface of nanoparticles. The results suggest that BSA encapsulated into polymers nanoparticles properly and investigated the stability of BSA NP formulations prepared by either double emulsion or microfluidic techniques.

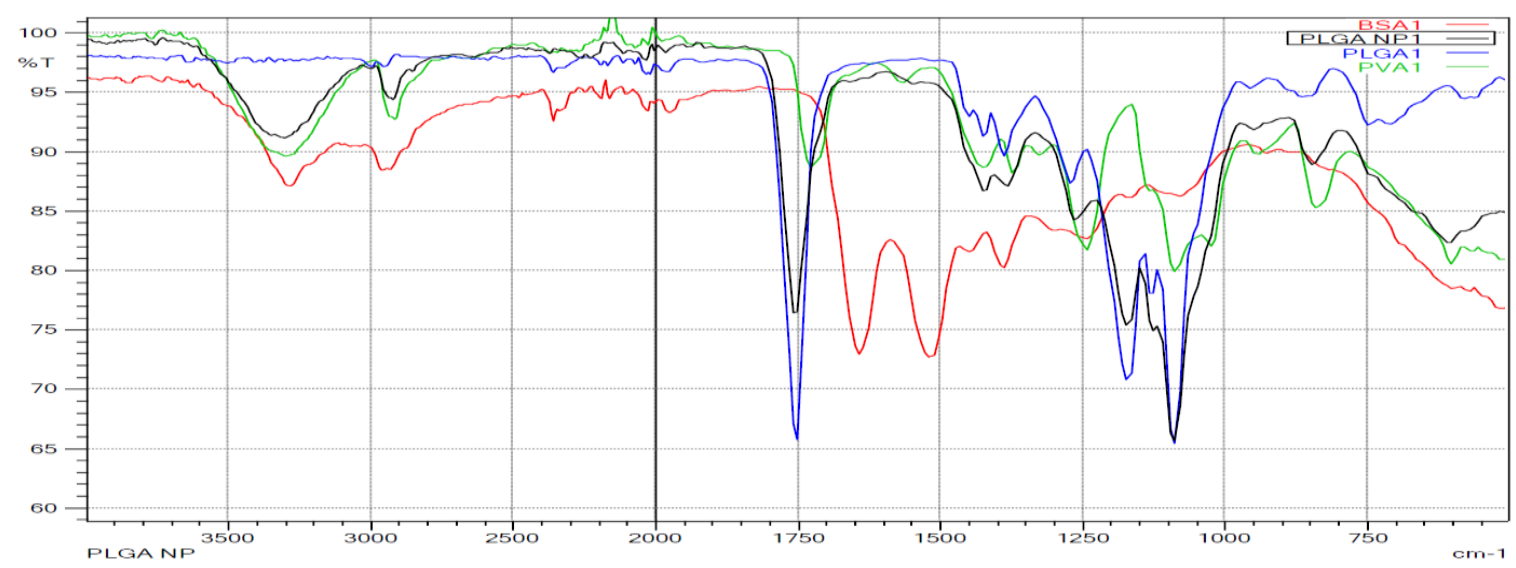

Figure (19): showing the FTIR spectrum of BSA loaded PLGA prepared by double emulsion BSA technique and their raw materials

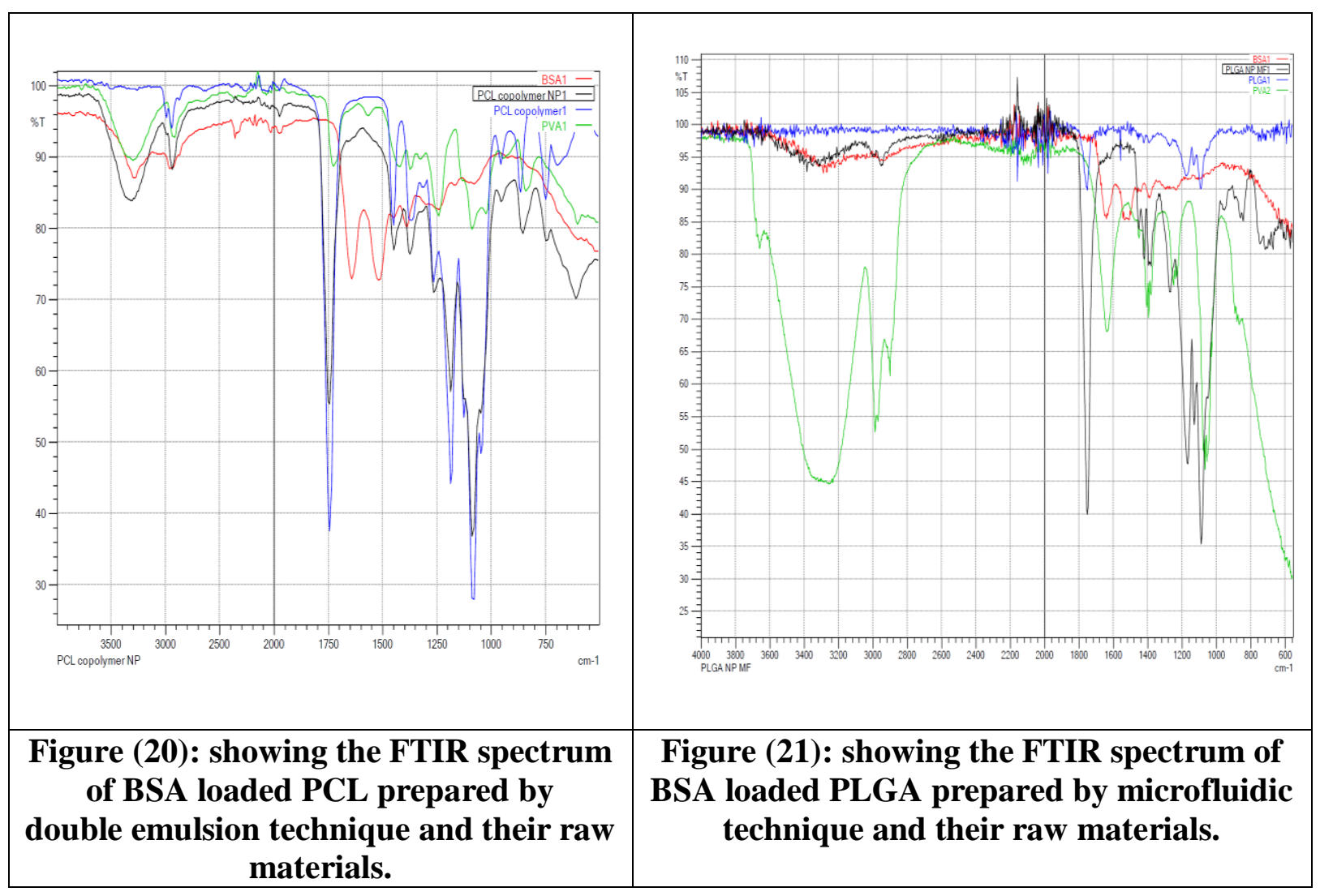




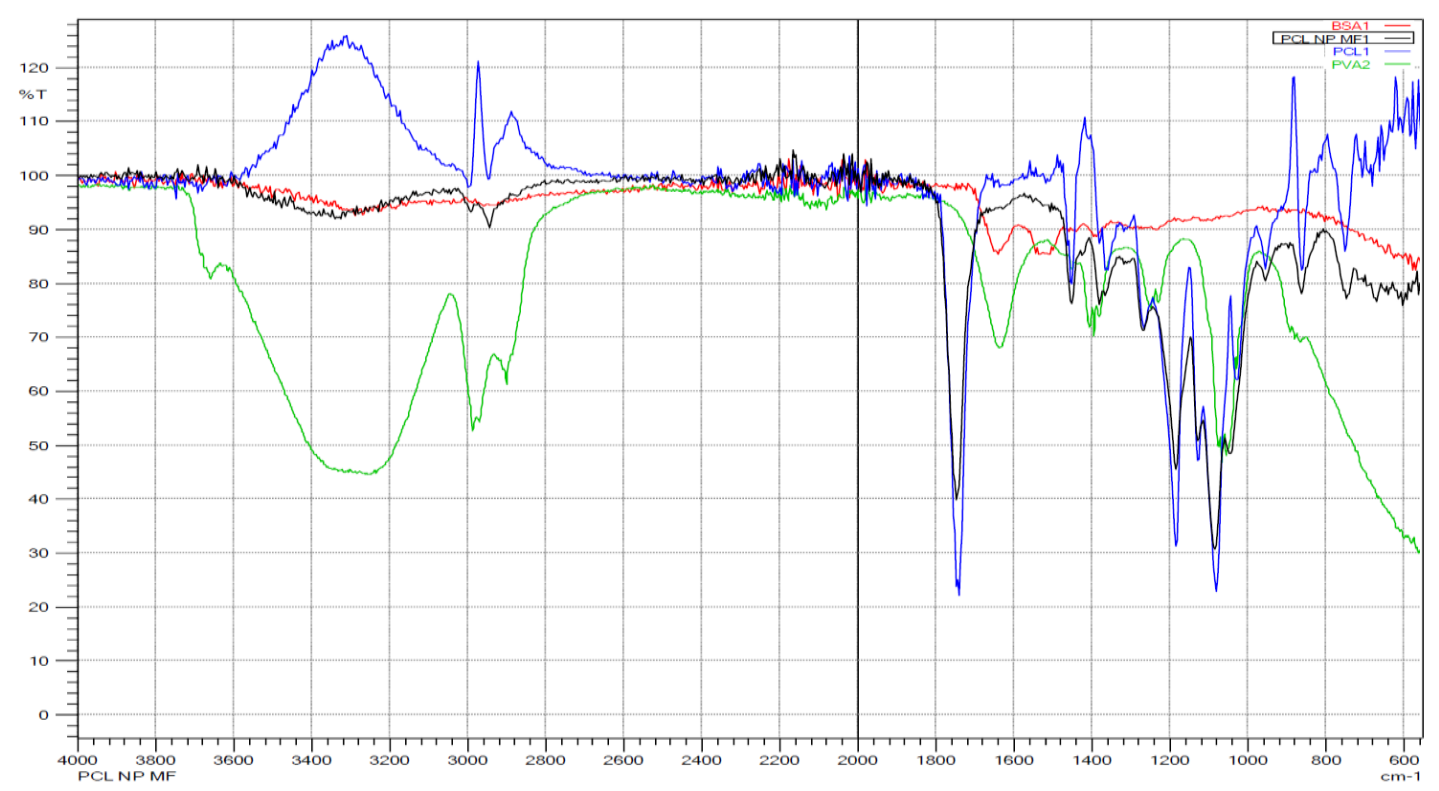

Figure (22): showing the FTIR spectrum of BSA loaded PCL prepared by microfluidic technique and their raw materials.

\section{Conclusion.}

Designing new formulations of biodegradable nanoparticles by achieving controlled release of peptide delivery provides more convenience for patients taking one dose rather than injecting multiple doses daily in a diabetic patient. The examination of nanoparticles obtained by using double emulsion technique / solvent evaporation or microfluidic technique was explained in this research. The bovine serum albumin (BSA) was used as a drug model and encapsulated through using two biodegradable polymers; PLGA (Poly D,L-lactic-co-glycolic acid) and Poly ( $\varepsilon$-caprolactone) (PCL).

The particles size obtained by microfluidic technique were smaller in size with lower poly dispersity index (PDI) than those particles size prepared by double emulsion technique. Therefore, the nanoparticles obtained by microfluidic technique had greater bioavailability, thus resulting in more therapeutic efficiency. BSA loaded PLGA had smaller particle size than BSA loaded PCL in both techniques. All formulations were in negative charge in range $7 \mathrm{mV}$. Furthermore, the encapsulation efficiency of nanoparticles obtained by microfluidic technique were greater than the encapsulation efficiency of nanoparticles obtained by double emulsion technique, which leads to decreased loss in the dose with nanoparticles obtained by microfluidic technique. In addition, the microfluidic technique improved the initial release on both polymers with shorter sustained release compared to the double emulsion technique, which suits drugs having narrow therapeutics window, which eventually leads to reducing the side effect. BSA loaded PLGA had higher initial burst with shorter sustained release than BSA loaded PCL. Moreover, after investigation the nanoparticles by scanning electron microscopy (SEM), the nanoparticles were spherical in shape in all formulations, which improves the bioavailability of drugs by increasing the surface area. The results confirm that particles size obtained by microfluidic technique had lower poly despersity index (PDI) compared to the particles size obtained by double emulsion technique, which improves the targeting capability towards specific organs and escaping from macrophages molecules. By using FTIR, the FTIR spectrums confirmed that BSA has been successfully encapsulated inside the biodegradable nanoparticles. This means that bovine serum albumin (BSA) as a 
drug model is protected by biodegradable polymers from harsh conditions such as $\mathrm{pH}$, temperature, and proteolysis by enzymes.

High encapsulation efficiency with high surface area of nanoparticle formulation leads to our objectives, which are controlled release design, escaping from macrophages cells to make longer sustained release, and protect from any harsh conditions. Therefore, the nanoparticles formulation with controlled release design helps several patients as diabetic mellitus patients requiring a maintained insulin level.

This study would form a foundation for researching aiming for developing various therapeutic peptides for targeting drug delivery and for pharmaceutic industries choosing suitable and effective methods for batch production.

\section{Suggestion for future work.}

More characterizations with regards to the physical and chemical stability of nanoparticles would be carried out such as DSC (Differential scanning calorimeter), because the only chemical stability was FTIR study in this research. DSC, which is a fundamental parameter, gives more details about thermal stability of nanoparticles and helps if the nanoparticles are stable or compatible with raw materials.

\section{References:}

1. Allahyari, M. and Mohit, E. (2015). Peptide/protein vaccine delivery system based on PLGA particles. Human Vaccines \&Immunotherapeutics, 12(3), pp.806-828.

2. Alibolandi, M., Alabdollah, F., Sadeghi, F., Mohammadi, M., Abnous, K., Ramezani, M. and Hadizadeh, F. (2016). Dextran-b-poly(lactide-co-glycolide) polymersome for oral delivery of insulin: In vitro and in vivo evaluation. Journal of Controlled Release, 227, pp. $58-70$

3. Chiesa, E., Dorati, R., Modena, T., Conti, B. and Genta, I. (2018). Multivariate analysis for the optimization of microfluidics-assisted nanoprecipitation method intended for the loading of small hydrophilic drugs into PLGA nanoparticles. International Journal of Pharmaceutics, 536(1), pp.165-177.

4. Coombes, A., Rizzi, S., Williamson, M., Barralet, J., Downes, S. and Wallace, W. (2004). Precipitation casting of polycaprolactone for applications in tissue engineering and drug delivery. Biomaterials, 25(2), pp.315-325.

5. Cohen-Sela, E., Chorny, M., Koroukhov, N., Danenberg, H. and Golomb, G. (2009). A new double emulsion solvent diffusion technique for encapsulating hydrophilic molecules in PLGA nanoparticles. Journal of Controlled Release, 133(2), pp.90-95.

6. Chu, L., Utada, A., Shah, R., Kim, J. and Weitz, D. (2007). Controllable Monodisperse Multiple Emulsions. AngewandteChemie International Edition, 46(47), pp.8970-8974.

7. Dinarvand, R., Sepehri, n., Manouchehri, Rouhani and Atyabi, F. (2011). Polylactide-coglycolide nanoparticles for controlled delivery of anticancer agents. International Journal of Nanomedicine, p.877.

8. Engineered Nanoparticles: Structure, Properties and Mechanisms of Toxicity Ashok K. Singh. (2017). MRS Bulletin, 42(01), p.75. 
9. Haggag, Y., Abdel-Wahab, Y., Ojo, O., Osman, M., El-Gizawy, S., El-Tanani, M., Faheem, A. and McCarron, P. (2016). Preparation and in vivo evaluation of insulinloaded biodegradable nanoparticles prepared from diblock copolymers of PLGA and PEG. International Journal of Pharmaceutics, 499(1-2), pp.236-246.

10. Hans, M. and Lowman, A. (2002). Biodegradable nanoparticles for drug delivery and targeting. Current Opinion in Solid State and Materials Science, 6(4), pp.319-327.

11. Iqbal, M., Zafar, N., Fessi, H. and Elaissari, A. (2018). Double emulsion solvent evaporation techniques used for drug encapsulation.

12. Iqbal, M., Zafar, N., Fessi, H. and Elaissari, A. (2015). Double emulsion solvent evaporation techniques used for drug encapsulation. International Journal of Pharmaceutics, 496(2), pp.173- 190.

13. Jain, R. (2000). The manufacturing techniques of various drug loaded biodegradable poly(lactide-co-glycolide) (PLGA) devices. Biomaterials, 21(23), pp.2475-2490.

14. Joshi, S., Hussain, M., Roces, C., Anderluzzi, G., Kastner, E., Salmaso, S., Kirby, D. and Perrie, Y. (2016). Microfluidics based manufacture of liposomes simultaneously entrapping hydrophilic and lipophilic drugs. International Journal of Pharmaceutics, 514(1), pp.160-168.

15. Kumari, A., Yadav, S. and Yadav, S. (2010). Biodegradable polymeric nanoparticles based drug delivery systems. Colloids and Surfaces B: Biointerfaces, 75(1), pp.1-18.

16. Martinez, N., Andrade, P., Durán, N. and Cavalitto, S. (2017). Development of double emulsion nanoparticles for the encapsulation of bovine serum albumin. Colloids and Surfaces B: Biointerfaces, 158, pp.190-196.

17. Mukherjee, B. (2008). Preparation, characterization and in-vitro evaluation of sustained release protein-loaded nanoparticles based on biodegradable polymers. International Journal of Nanomedicine, p.487.

18. Morishita, M. and Peppas, N. (2006). Is the oral route possible for peptide and protein drug delivery?. Drug Discovery Today, 11(19-20), pp.905-910.

19. Lassalle, V. and Ferreira, M. (2010). PLGA based drug delivery systems (DDS) for the sustained release of insulin: insight into the protein/polyester interactions and the insulin release behavior. Journal of Chemical Technology \& Biotechnology, 85(12), pp.15881596.

20. Li, X. (1999). Investigation on process parameters involved in preparation of poly-?lactide-poly(ethylene glycol) microspheres containing LeptospiraInterrogans antigens. International Journal of Pharmaceutics, 178(2), pp.245-255.

21. Locatelli, E. and Comes Franchini, M. (2012). Biodegradable PLGA-b-PEG polymeric nanoparticles: synthesis, properties, and nanomedical applications as drug delivery system. Journal of Nanoparticle Research, 14(12). 
22. Pathak, Y. and Thassu, D. (2009). Drug delivery nanoparticles formulation and characterization. 1st ed. New York: Informa Healthcare.

23. Park, T. (1995). Degradation of poly(lactic-co-glycolic acid) microspheres: effect of copolymer composition. Biomaterials, 16(15), pp.1123-1130.

24. Patel, A., Patel, M., Yang, X. and Mitra, A. (2014). Recent Advances in Protein and Peptide Drug Delivery: A Special Emphasis on Polymeric Nanoparticles. Protein \& Peptide Letters, 21(11), pp.1102-1120.

25. Pessi, J., Santos, H., Miroshnyk, I., JoukoYliruusi, Weitz, D. and Mirza, S. (2014). Microfluidics-assisted engineering of polymeric microcapsules with high encapsulation efficiency for protein drug delivery. International Journal of Pharmaceutics, 472(1-2), pp.82- 87.

26. Precisionnanosystems.com. (2018). Precision NanoSystems - Create Transformative Medicines. [online] Available at: https://www.precisionnanosystems.com/ [Accessed 6 Sep. 2018].

27. Sahoo, S., Panyam, J., Prabha, S. and Labhasetwar, V. (2002). Residual polyvinyl alcohol associated with poly (d,l-lactide-co-glycolide) nanoparticles affects their physical properties and cellular uptake. Journal of Controlled Release, 82(1), pp.105-114.

28. Schliecker, G., Schmidt, C., Fuchs, S. and Kissel, T. (2003). Characterization of a homologous series of d , 1 -lactic acid oligomers; a mechanistic study on the degradation kinetics in vitro. Biomaterials, 24(21), pp.3835-3844.

29. Tavares, M., Menezes, L., Dutra Filho, J., Cabral, L. and Tavares, M. (2017). Surfacecoated polycaprolactone nanoparticles with pharmaceutical application: Structural and molecular mobility evaluation by TD-NMR. Polymer Testing, 60, pp.39-48.

30. Thermofisher.com. (2018). Thermo Fisher Scientific - UK. [online] Available at: https://www.thermofisher.com [Accessed 6 Sep. 2018].

31. Wang, W., Zhang, M. and Chu, L. (2014). Microfluidic approach for encapsulation via double emulsions. Current Opinion in Pharmacology, 18, pp.35-41.

32. Woodruff, M. and Hutmacher, D. (2010). The return of a forgotten polymerPolycaprolactone in the 21st century. Progress in Polymer Science, 35(10), pp.1217-1256.

33. Xiang, S., Scholzen, A., Minigo, G., David, C., Apostolopoulos, V., Mottram, P. and Plebanski, M. (2006). Pathogen recognition and development of particulate vaccines: Does size matter?. Methods, 40(1), pp.1-9

34. Yeo, Y. and Park, K. (2004). Control of encapsulation efficiency and initial burst in polymeric microparticle systems. Archives of Pharmacal Research, 27(1), pp.1-12.

35. Zambaux, M. (1998). Influence of experimental parameters on the characteristics of poly(lactic acid) nanoparticles prepared by a double emulsion method. Journal of Controlled Release, 50(1-3), pp.31-40. 